\title{
Bubbling analysis for approximate Lorentzian harmonic maps from Riemann surfaces
}

\author{
Xiaoli Han ${ }^{1} \cdot$ Jürgen Jost $^{2} \cdot$ Lei Liu $^{2} \cdot$ Liang Zhao $^{3}$
}

Received: 3 May 2017 / Accepted: 25 October 2017 / Published online: 7 November 2017

(C) The Author(s) 2017. This article is an open access publication

\begin{abstract}
For a sequence of approximate harmonic maps $\left(u_{n}, v_{n}\right)$ (meaning that they satisfy the harmonic system up to controlled error terms) from a compact Riemann surface with smooth boundary to a standard static Lorentzian manifold with bounded energy, we prove that identities for the Lorentzian energy hold during the blow-up process. In particular, in the special case where the Lorentzian target metric is of the form $g_{N}-\beta d t^{2}$ for some Riemannian metric $g_{N}$ and some positive function $\beta$ on $N$, we prove that such identities also hold for the positive energy (obtained by changing the sign of the negative part of the Lorentzian energy) and there is no neck between the limit map and the bubbles. As an application, we complete the blow-up picture of singularities for a harmonic map flow into a standard static Lorentzian manifold. We prove that the energy identities of the flow hold at both finite and infinite singular times. Moreover, the no neck property of the flow at infinite singular time is true.
\end{abstract}

\section{Mathematics Subject Classification $53 \mathrm{C} 43 \cdot 58 \mathrm{E} 20$}

Communicated by Luigi Ambrosio.

The research is supported by NSFC Nos. 11471014, 11471299 and the Fundamental Research Funds for the Central Universities. The authors would like to thank the referee for detailed and useful comments.

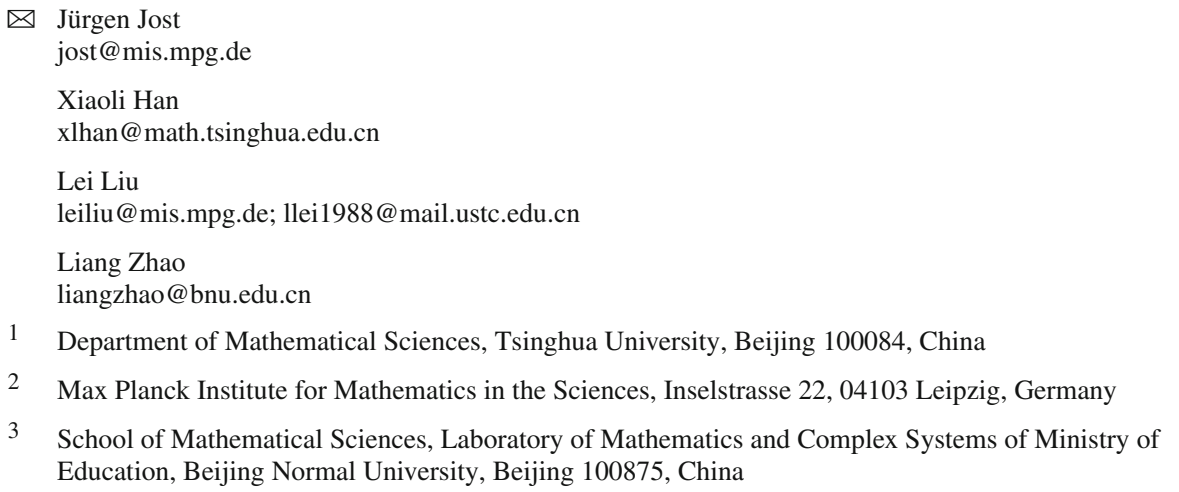




\section{Introduction}

Harmonic maps constitute one of the fundamental objects in the field of geometric analysis. When the domain is two-dimensional, particularly interesting features arise. The conformal invariance of the energy functional leads to non-compactness of the set of harmonic maps in dimension two, and the blow-up behavior has been studied extensively in [5, 13, 20, 23, 24, 27] for the interior case and $[10,15,16]$ for the boundary case. Roughly speaking, the energy identities for harmonic maps tell us that, during the weak convergence of a sequence of harmonic maps, the loss of energy is concentrated at finitely many points and can be quantized by a sum of energies of harmonic spheres and harmonic disks. Also for many other elliptic and parabolic nonlinear variational problems arising in geometry and physics, such as Jholomorphic curves or Yang-Mills fields, to understand the convergence properties of a sequence and the emergence of singularities is of special importance.

In physics, harmonic maps arise as a mathematical representation of the nonlinear sigma model and this leads to several generalizations. For example, motivated by the supersymmetric sigma model, Dirac harmonic maps where a map is coupled with a spinor field have been extensively studied. One can refer to $[4,14,31]$ and the references therein. From the perspective of general relativity, it is also natural to generalize the target of a harmonic map to a Lorentzian manifold. Recent work on minimal surfaces in anti-de-Sitter space and their applications in theoretical physics (see e.g. Alday and Maldacena [1]) shows the importance of this extension. Geometrically, the link between harmonic maps into $\mathbb{S}_{1}^{4}$ and the conformal Gauss maps of Willmore surfaces in $\mathbb{S}^{3}$ [3] also naturally leads to such harmonic maps.

Thus, in this paper, we investigate harmonic maps from Riemann surfaces into Lorentzian manifolds. In order to gain some special structure, we consider a Lorentzian manifold $N \times \mathbb{R}$ that is equipped with a warped product metric of the form

$$
g=g_{N}-\beta(d \theta+\omega)^{2},
$$

where $\left(\mathbb{R}, d \theta^{2}\right)$ is the 1-dimensional Euclidean space, $\left(N, g_{N}\right)$ is an $n$-dimensional compact Riemannian manifold which by Nash's theorem can be isometrically embedded into some $\mathbb{R}^{K}, \beta$ is a positive $C^{\infty}$ function on $N$ and $\omega$ is a smooth 1 -form on $N$. Since $N$ is compact, $\beta$ and $\omega$ are both bounded on $N$. We suppose for any $p \in N$,

$$
0<\lambda_{1}<\beta(p)<\lambda_{2},|\omega(p)|+|\nabla \omega(p)|+|\nabla \beta(p)| \leq \lambda_{2} .
$$

A Lorentzian manifold with a metric of the form (1.1) is called a standard static manifold. For more details on such manifolds, we refer to [17,22].

Let $(M, h)$ be a compact Riemann surface with smooth boundary $\partial M$. For a map $(u, v) \in$ $C^{2}(M, N \times \mathbb{R})$ with fixed boundary data $\left.(u, v)\right|_{\partial M}=(\phi, \psi)$, we define the functional

$$
E_{g}(u, v)=\frac{1}{2} \int_{M}\left\{|\nabla u|^{2}-\beta(u)\left|\nabla v+\omega_{i}(u) \nabla u^{i}\right|^{2}\right\} d v_{h},
$$

which is called the Lorentzian energy of the map $(u, v)$ on $M$. Critical points $(u, v)$ in $C^{2}(M, N \times \mathbb{R})$ of the functional (1.2) are called Lorentzian harmonic maps from $(M, h)$ into the Lorentzian manifold $(N \times \mathbb{R}, g)$. Besides the Lorentzian energy $E_{g}(u, v)$, we also consider

$$
E(u)=\frac{1}{2} \int_{M}|\nabla u|^{2} d v_{h} \text { and } E(u, v)=\frac{1}{2} \int_{M}\left\{|\nabla u|^{2}+|\nabla v|^{2}\right\} d v_{h}
$$

and call it the positive energy of $u$ and $(u, v)$ on $M$ respectively. It is obvious that both the Lorentzian and positive energy functionals are conformally invariant when $\operatorname{dim} M=2$. 
Zhu [32] has derived the Euler-Lagrange equations for (1.2),

$$
\begin{gathered}
\Delta u+A(u)(\nabla u, \nabla u)-H^{\top}=0 \text { in } M, \\
\operatorname{div}\left(\beta(u)\left(\nabla v+\omega_{i} \nabla u^{i}\right)\right)=0 \text { in } M
\end{gathered}
$$

with the boundary data

$$
\left.(u, v)\right|_{\partial M}=(\phi, \psi) \in C^{2+\alpha}(\partial M, N \times \mathbb{R})
$$

for some $\alpha \in(0,1)$. Here $A$ is the second fundamental form of $N$ in $\mathbb{R}^{K}, H^{\top}$ is the tangential part of $H=\left(H^{1}, \ldots, H^{K}\right)$ along the map $(u, v)$ with

$$
H^{j}:=\beta\left(\nabla v+\omega_{i} \nabla u^{i}\right) \cdot \nabla u^{k}\left(\frac{\partial \omega_{j}}{\partial y^{k}}-\frac{\partial \omega_{k}}{\partial y^{j}}\right)-\frac{1}{2} \frac{\partial \beta}{\partial y^{j}}\left|\nabla v+\omega_{i} \nabla u^{i}\right|^{2}, j=1, \ldots, K .
$$

Let us now recall some related results. The existence of geodesics in Lorentzian manifolds was studied in [2]. Variational methods for such harmonic maps were developed in [6,7]. Recently, [8] studied the corresponding heat flow under the assumption that $\omega \equiv 0$ and proved the existence of a Lorentzian harmonic map in any given homotopic class under either some geometric conditions on $N$ or a small energy condition of the initial maps. The regularity theory of Lorentzian harmonic maps was studied in [11,12,19,32].

In [9], the authors proved identities of the Lorentzian energy for a blow-up sequence of Lorentzian harmonic maps when $M$ is a compact Riemann surface without boundary. They showed the tangential Lorentzian energy of the sequence in the neck region has no concentration by comparing the energy with piece-wise linear functions (i.e. geodesics). Then they used the Hopf differentials to control the radial Lorentzian energy.

In any case, the analysis of Lorentzian harmonic maps is more difficult than that of standard (Riemannian) harmonic maps, because one cannot no longer use positivity properties of the target metric. This is a technical reason why we restrict ourselves to standard static Lorentzian manifolds.

In this paper, we shall prove some energy identities of an approximate Lorentzian harmonic map sequence and get the no neck property during a blow-up process when $M$ is a compact Riemann surface with boundary. We work with approximate sequences which means that we allow for error terms in the Lorentzian harmonic maps system. The reason is that this has a direct application in studying the singularities of the parabolic version, the Lorentzian harmonic map flow (see [8]). Moreover, since we assume that the domain $M$ is a manifold with boundary, blow-up analysis on the boundary must be included in our case. Here, we will use the method of integrating by parts (cf. [20] for harmonic maps) to prove a Pohozaev type identity instead of using the Hopf differential. The Pohozaev identity method is more general and powerful than the Hopf differential method. We first prove identities for the Lorentzian energy for a blow-up sequence of approximate Lorentzian harmonic maps. Furthermore, for the special case $\omega \equiv 0$, we show that also such identities for the positive energy and no neck properties hold.

Throughout this paper, we call a map into $N \times \mathbb{R}$ a Lorentzian map and when we have a map into the Riemannian manifold $N$, we just call it a map. We first give the definition of an approximate Lorentzian harmonic map.

Definition 1.1 $(u, v) \in W^{2,2}(M, N \times \mathbb{R})$ is called an approximate Lorentzian harmonic map with Dirichlet boundary data $(\phi, \psi)$, if there exist fields $(\tau(u, v), \kappa(u, v)) \in L^{1}(M)$ such that $(u, v)$ satisfies 


$$
\begin{gathered}
\Delta u+A(u)(\nabla u, \nabla u)-H^{\top}=\tau(u, v) \text { in } M, \\
\operatorname{div}\left(\beta(u)\left(\nabla v+\omega_{i} \nabla u^{i}\right)\right)=\kappa(u, v) \text { in } M
\end{gathered}
$$

with the boundary condition $\left.(u, v)\right|_{\partial M}=(\phi, \psi)$.

Now we can present our first main result.

Theorem 1.1 Let $\left(u_{n}, v_{n}\right) \in W^{2,2}(M, N \times \mathbb{R})$ be a sequence of approximate harmonic maps with Dirichlet boundary $\left.\left(u_{n}, v_{n}\right)\right|_{\partial M}=(\phi, \psi) \in C^{2+\alpha}(\partial M, N \times \mathbb{R})$ satisfying

$$
E\left(u_{n}, v_{n}\right)+\left\|\left(\tau_{n}, \kappa_{n}\right)\right\|_{L^{2}(M)} \leq \Lambda<\infty
$$

where $\left\|\left(\tau_{n}, \kappa_{n}\right)\right\|_{L^{2}(M)}^{2}=\left\|\tau_{n}\right\|_{L^{2}(M)}^{2}+\left\|\kappa_{n}\right\|_{L^{2}(M)}^{2}$. After taking a subsequence, still denoted by $\left\{u_{n}, v_{n}\right\}$, we can find a finite set $\mathcal{S}=\left\{p_{1}, \ldots, p_{I}\right\}$ and a limitmap $\left(u_{0}, v_{0}\right) \in W^{1,2}(M, N \times \mathbb{R})$ with Dirichlet boundary data $\left.\left(u_{0}, v_{0}\right)\right|_{\partial M}=(\phi, \psi)$ such that $\left\{\left(u_{n}, v_{n}\right)\right\}$ converges weakly in $W_{\text {loc }}^{2,2}(M \backslash \mathcal{S})$ to $\left(u_{0}, v_{0}\right)$. Moreover, there are finitely many nontrivial Lorentzian harmonic spheres $\left(\sigma_{i}^{l}, \xi_{i}^{l}\right): \mathbb{S}^{2} \rightarrow N \times \mathbb{R}$ and nontrivial Lorentzian harmonic maps $\left(\sigma_{i}^{k}, \xi_{i}^{k}\right): \mathbb{R}_{+}^{2}:=$ $\left\{\left(x^{1}, x^{2}\right) \in \mathbb{R}^{2} \mid x^{2} \geq 0\right\} \rightarrow N \times \mathbb{R}$ with constant boundary values, where $i=1, \ldots, I$, $l=1, \ldots, l_{i}$ and $k=1, \ldots, k_{i}$ with $l_{i}, k_{i} \geq 0$ and $l_{i}+k_{i} \geq 1$, such that

$$
\lim _{n \rightarrow \infty} E_{g}\left(u_{n}, v_{n}\right)=E_{g}\left(u_{0}, v_{0}\right)+\sum_{i=1}^{I} \sum_{l=1}^{l_{i}} E_{g}\left(\sigma_{i}^{l}, \xi_{i}^{l}\right)+\sum_{i=1}^{I} \sum_{k=1}^{k_{i}} E_{g}\left(\sigma_{i}^{k}, \xi_{i}^{k}\right) .
$$

Here and in the sequel, "finite" includes "possibly empty", that is, singularities need not always arise. Since this is obvious, it will not be explicitly mentioned.

When $\omega \equiv 0$, the equations for Lorentzian harmonic maps become

$$
\begin{aligned}
\Delta u+A(u)(\nabla u, \nabla u)-B^{\top}(u)|\nabla v|^{2} & =0 \text { in } M, \\
\operatorname{div}(\beta(u) \nabla v) & =0 \text { in } M
\end{aligned}
$$

where $B(u):=\left(B^{1}, B^{2}, \ldots, B^{K}\right)$ with

$$
B^{j}:=-\frac{1}{2} \frac{\partial \beta(u)}{\partial y^{j}}
$$

and $B^{\top}$ is the tangential part of $B$ along the map $u$. In this case, the blow-up behavior is simpler. We show that the identities for the positive energy hold and there is no neck during the process.

Theorem 1.2 If we additionally assume $\omega \equiv 0$ in Theorem 1.1, there cannot emerge any Lorentzian harmonic maps $\left(\sigma_{i}^{k}, \xi_{i}^{k}\right): \mathbb{R}_{+}^{2}:=\left\{\left(x^{1}, x^{2}\right) \in \mathbb{R}^{2} \mid x^{2} \geq 0\right\} \rightarrow N \times \mathbb{R}$ during the blow-up process [i.e. $k_{i}=0$ in (1.9)]. Moreover, the components $\xi_{i}^{l}$ of the maps $\left(\sigma_{i}^{l}, \xi_{i}^{l}\right)$ are constant and $\sigma_{i}^{l}: \mathbb{S}^{2} \rightarrow N, 1 \leq l \leq l_{i}$ are nontrivial harmonic spheres. In this case, (1.9) becomes

$$
\begin{aligned}
& \lim _{n \rightarrow \infty} E\left(u_{n}\right)=E\left(u_{0}\right)+\sum_{i=1}^{I} \sum_{l=1}^{l_{i}} E\left(\sigma_{i}^{l}\right), \\
& \lim _{n \rightarrow \infty} E\left(v_{n}\right)=E\left(v_{0}\right) .
\end{aligned}
$$

and the image $u_{0}(M) \cup_{i=1}^{I} \cup_{l=1}^{l_{i}}\left(\sigma_{i}^{l}\left(\mathbb{S}^{2}\right)\right)$ is a connected set in $N$. 
As an application of Theorem 1.2, we consider a harmonic map heat flow

$$
\begin{cases}\partial_{t} u=\Delta u+A(u)(\nabla u, \nabla u)-B^{\top}(u)|\nabla v|^{2}, & \text { in } M \times[0, T) \\ -\operatorname{div}(\beta(u) \nabla v)=0, & \text { in } M \times[0, T)\end{cases}
$$

with the boundary-initial data

$$
\begin{cases}u(x, t)=\phi_{0}(x) & \text { on } M \times\{t=0\}, \\ u(x, t)=\phi(x), & \text { on } \partial M \times\{t>0\}, \\ v(x, t)=\psi(x), & \text { on } \partial M \times\{t>0\}, \\ \phi_{0}(x)=\phi(x) & \text { on } \partial M .\end{cases}
$$

This kind of harmonic map heat flow is a parabolic-elliptic system and was first studied in [8]. We proved the problem (1.14) and (1.15) admits a unique solution $(u, v) \in \mathcal{V}\left(M_{0}^{T_{1}} ; N \times \mathbb{R}\right)$ (see the notation at the end of this section), where $T_{1}$ is the first singular time and some bubbles (nontrivial harmonic spheres) split off at $t=T_{1}$. In this paper, we complete the blow-up picture at the singularities of this flow. First, we have

Theorem 1.3 Suppose the problem (1.14) and (1.15) admits a unique global solution $(u, v) \in \mathcal{V}\left(M_{0}^{\infty} ; N \times \mathbb{R}\right)$ which blows up at infinity, i.e. $T_{1}=\infty$. By choosing some $t_{n} \rightarrow \infty$, there exist a smooth Lorentzian harmonic map $\left(u_{\infty}, v_{\infty}\right): M \rightarrow N \times \mathbb{R}$ with boundary data $\left.\left(u_{\infty}, v_{\infty}\right)\right|_{\partial M}=(\phi, \psi)$ and finitely many harmonic spheres $\left\{\sigma^{i}\right\}_{i=1}^{L}: \mathbb{R}^{2} \cup\{\infty\} \rightarrow N$ such that

$$
\begin{gathered}
\lim _{n \rightarrow \infty} E\left(u\left(t_{n}\right) ; M\right)=E\left(u_{\infty}, M\right)+\sum_{i=1}^{L} E\left(\sigma^{i}\right), \\
\lim _{t \rightarrow \infty} E_{g}(u(t), v(t) ; M)=E_{g}\left(u_{\infty}, v_{\infty} ; M\right)+\sum_{i=1}^{L} E\left(\sigma^{i}\right) .
\end{gathered}
$$

Furthermore, there exist sequences $\left\{x_{n}^{i}\right\}_{i=1}^{L} \subset M$ and $\left\{r_{n}^{i}\right\}_{i=1}^{L} \subset \mathbb{R}_{+}$such that

$$
\lim _{n \rightarrow \infty}\left\|u\left(\cdot, t_{n}\right)-u_{\infty}(\cdot)-\sum_{i=1}^{L} \sigma_{n}^{i}(\cdot)\right\|_{L^{\infty}(M)}=0,
$$

where $\sigma_{n}^{i}(\cdot)=\sigma^{i}\left(\frac{-x_{n}^{i}}{r_{n}^{i}}\right)-\sigma_{i}(\infty)$.

When the flow blows up at finite time, we have

Theorem 1.4 Let $(u, v) \in \mathcal{V}\left(M_{0}^{T_{1}} ; N \times \mathbb{R}\right)$ be a solution to $(1.14)$ and (1.15) with $T_{1}<\infty$ as its first singular time. Then there exist finitely many harmonic spheres $\left\{\sigma^{i}\right\}_{i=1}^{L}: \mathbb{R}^{2} \cup\{\infty\} \rightarrow$ $N$ such that

$$
\begin{gathered}
\lim _{t \nearrow T_{1}} E(u(t) ; M)=E\left(u\left(T_{1}\right), M\right)+\sum_{i=1}^{L} E\left(\sigma^{i}\right), \\
\lim _{t \nearrow T_{1}} E_{g}(u(t), v(t) ; M)=E_{g}\left(u\left(T_{1}\right), v\left(T_{1}\right) ; M\right)+\sum_{i=1}^{L} E\left(\sigma^{i}\right),
\end{gathered}
$$

where $\left(u\left(T_{1}\right), v\left(T_{1}\right)\right)$ is the weak limit of $(u(t), v(t))$ in $W^{1,2}(M)$ as $t \rightarrow T_{1}$. 
The paper is organized as follows. In Sect. 2, we derive some basic lemmas including the small energy regularity, a Pohozaev type identity and a removable singularity result. In Sect. 3, we prove the energy identities and no neck property for a sequence of approximate Lorentzian harmonic maps (Theorems 1.1, 1.2). In Sect. 4, we apply these two results to the harmonic map heat flow and prove Theorems 1.3 and 1.4. Throughout this paper, we use $C$ to denote a universal constant and denote $D_{1}(0):=\left\{\left.(x, y) \in \mathbb{R}^{2}|| x\right|^{2}+|y|^{2} \leq 1\right\}$, $D_{1}^{+}(0):=\left\{\left.(x, y) \in \mathbb{R}^{2}|| x\right|^{2}+|y|^{2} \leq 1, y \geq 0\right\}, \partial^{0} D_{1}^{+}(0):=\left\{(x, y) \in D_{1}^{+}(0) \mid y=0\right\}$ and $\partial^{+} D_{1}^{+}(0):=\left\{(x, y) \in \partial D_{1}^{+}(0) \mid y>0\right\}$.

Notation We denote

$$
\begin{aligned}
\mathcal{V}\left(M_{s}^{t} ; N \times \mathbb{R}\right):= & \left\{(u, v): M \times[s, t) \rightarrow N \times \mathbb{R}, v \in L^{\infty}\left([s, t) ; C^{2+\alpha}(M)\right),\right. \\
& v, \nabla v \in \cap_{s<\rho<t} C^{\alpha, \alpha / 2}(M \times[s, \rho]), \\
& \left.u \in \cap_{s<\rho<t} C^{2+\alpha, 1+\alpha / 2}(M \times[s, \rho])\right\} .
\end{aligned}
$$

\section{2 some basic lemmas}

In this section, we will prove some basic lemmas for Lorentzian harmonic maps, such as the small energy regularity, a Pohozaev type identity and a removable singularity result.

First, we present two small energy regularity lemmas corresponding to the interior case and the boundary case. For harmonic maps, such results have been obtained in [5,27] for the interior case and in $[10,15,16]$ for the boundary case. We use

$$
\bar{u}_{\Omega}=\frac{1}{|\Omega|} \int_{\Omega} u d x
$$

to denote the average value of a function $u$ on the domain $\Omega$. Here and in the sequel, we shall view $(\phi, \psi)$ as the restriction of some $C^{2+\alpha}(M, N \times \mathbb{R})$ map on $\partial M$ and for simplicity, we still denote it by $(\phi, \psi)$.

Lemma 2.1 Let $(u, v) \in W^{2, p}(D, N \times \mathbb{R}), 1<p \leq 2$ be an approximate Lorentzian harmonic map with $(\tau, \kappa) \in L^{p}(D)$. There exist constants $\epsilon_{1}=\epsilon_{1}\left(p, \lambda_{1}, \lambda_{2}, N\right)>0$ and $C=C\left(p, \lambda_{1}, \lambda_{2}, N\right)>0$, such that if $E(u, v ; D) \leq \epsilon_{1}$, we have

$$
\begin{aligned}
& \left\|u-\bar{u}_{D_{1 / 2}}\right\|_{W^{2, p}\left(D_{1 / 2}\right)}+\left\|v-\bar{v}_{D_{1 / 2}}\right\|_{W^{2, p}\left(D_{1 / 2}\right)} \\
& \quad \leq C\left(\|\nabla u\|_{L^{2}(D)}+\|\nabla v\|_{L^{2}(D)}+\|\tau\|_{L^{p}(D)}+\|\kappa\|_{L^{p}(D)}\right) .
\end{aligned}
$$

Moreover, by the Sobolev embedding $W^{2, p} \hookrightarrow C^{0}$, we have

$$
\|u\|_{o s c\left(D_{1 / 2}\right)}=\sup _{x, y \in D_{1 / 2}}|u(x)-u(y)| \leq C\left(\|(\nabla u, \nabla v)\|_{L^{2}(D)}+\|(\tau, \kappa)\|_{L^{p}(D)}\right) .
$$

For the boundary case, we have

Lemma 2.2 Let $(u, v) \in W^{2, p}\left(D^{+}, N \times \mathbb{R}\right), 1<p \leq 2$ be an approximate Lorentzian harmonic map with $(\tau, \kappa) \in L^{p}\left(D^{+}\right)$. On the boundary we assume that $\left.u\right|_{\partial^{0} D^{+}}=\phi(x)$ and $\left.v\right|_{\partial^{0} D^{+}}=\psi(x)$ where $(\phi, \psi) \in C^{2+\alpha}(D)$. There exist constants $\epsilon_{2}=\epsilon_{2}\left(p, \lambda_{1}, \lambda_{2}, N\right)>0$ and $C=C\left(p, \lambda_{1}, \lambda_{2}, N\right)>0$, such that if $E\left(u, v ; D_{1}^{+}\right) \leq \epsilon_{2}$, we have

$$
\begin{aligned}
& \left\|u-\bar{\phi}_{\partial^{0} D^{+}}\right\|_{W^{2, p}\left(D_{1 / 2}^{+}\right)}+\left\|v-\bar{\psi}_{\partial^{0} D^{+}}\right\|_{W^{2, p}\left(D_{1 / 2}^{+}\right)} \\
& \leq C\left(\|(\nabla u, \nabla v)\|_{L^{2}\left(D^{+}\right)}+\|(\nabla \phi, \nabla \psi)\|_{W^{1, p}\left(D^{+}\right)}+\|(\tau, \kappa)\|_{L^{p}\left(D^{+}\right)}\right),
\end{aligned}
$$


where $\bar{\phi}_{\partial^{0} D^{+}}=\frac{1}{2} \int_{\partial^{0} D_{1}^{+}} \phi d x$ and $\bar{\psi}_{\partial^{0} D^{+}}=\frac{1}{2} \int_{\partial^{0} D_{1}^{+}} \psi d x$.

Moreover, by the Sobolev embedding $W^{2, p} \hookrightarrow C^{0}$, we have

$$
\begin{aligned}
\|u\|_{o s c\left(D_{1 / 2}^{+}\right)} & =\sup _{x, y \in D_{1 / 2}^{+}}|u(x)-u(y)| \\
& \leq C\left(\|(\nabla u, \nabla v)\|_{L^{2}\left(D^{+}\right)}+\|(\nabla \phi, \nabla \psi)\|_{W^{1, p}\left(D^{+}\right)}+\|(\tau, \kappa)\|_{L^{p}\left(D^{+}\right)}\right) .
\end{aligned}
$$

Since the proof of the interior case is similar to and simpler than that of the boundary case, we only prove Lemma 2.2 and omit the proof of Lemma 2.1.

Proof Without loss of generality, we assume $\bar{\phi}_{\partial^{0} D^{+}}=\bar{\psi}_{\partial^{0} D^{+}}=0$. Choosing a cut-off function $\eta \in C_{0}^{\infty}\left(D^{+}\right)$satisfying $0 \leq \eta \leq 1,\left.\eta\right|_{D_{3 / 4}^{+}} \equiv 1,|\nabla \eta|+\left|\nabla^{2} \eta\right| \leq C$ and computing directly, we get

$$
\begin{aligned}
|\Delta(\eta u)|= & |\eta \Delta u+2 \nabla \eta \nabla u+u \Delta \eta| \\
\leq & C(|u|+|\nabla u|+(|\nabla u|+|\nabla v|)(|\eta \nabla u|+|\eta \nabla v|)+|\tau|) \\
\leq & C(|\nabla u|+|\nabla v|)(|\nabla(\eta u)|+|\nabla(\eta v)|) \\
& +C(|u|+(1+|v|)(|\nabla u|+|\nabla v|)+|\tau|) .
\end{aligned}
$$

Similarly,

$$
\begin{aligned}
|\Delta(\eta v)|= & |\eta \Delta v+2 \nabla \eta \nabla v+v \Delta \eta| \\
\leq & C(|v|+|\nabla v|+(|\nabla u|+|\nabla v|)(|\eta \nabla u|+|\eta \nabla v|)+|\tau|+|\kappa|) \\
\leq & C(|\nabla u|+|\nabla v|)(|\nabla(\eta u)|+|\nabla(\eta v)|) \\
& +C(|v|+(1+|v|)(|\nabla u|+|\nabla v|)+|\tau|+|\kappa|) .
\end{aligned}
$$

First we assume that $1<p<2$. By standard elliptic estimates and Poincare's inequality, we obtain

$$
\begin{aligned}
\|\eta u\|_{W^{2, p}(D)}+\|\eta v\|_{W^{2, p}(D)} & \leq C\|(\nabla u, \nabla v)\|_{L^{2}\left(D^{+}\right)}\|(\nabla(\eta u), \nabla(\eta v))\|_{L^{\frac{2 p}{2-p}}\left(D^{+}\right)}+C\|(u, v)\|_{W^{1, p}\left(D^{+}\right)} \\
& +C\left(\|(\nabla u, \nabla v)\|_{L^{2}\left(D^{+}\right)}\|v\|_{L^{\frac{2 p}{2-p}\left(D^{+}\right)}}+\||(\phi, \psi)|\|_{W^{2, p}\left(D^{+}\right)}+\||(\tau, \kappa)|\|_{L^{p}\left(D^{+}\right)}\right) \\
& \leq C \epsilon_{2}\|(\nabla(\eta u), \nabla(\eta v))\|_{L^{\frac{2 p}{2-p}}\left(D^{+}\right)}+C\left(\|(\nabla u, \nabla v)\|_{L^{2}\left(D^{+}\right)}+\||(\nabla \phi, \nabla \psi)|\|_{W^{1, p}\left(D^{+}\right)}\right. \\
& \left.+\||(\tau, \kappa)|\|_{L^{p}\left(D^{+}\right)}\right),
\end{aligned}
$$

where we use the Sobolev inequality

$$
\|v\|_{L^{\frac{2 p}{2-p}\left(D^{+}\right)}} \leq C(p)\|\nabla v\|_{L^{2}\left(D^{+}\right)} .
$$

Taking $\epsilon_{2}>0$ sufficiently small, we have

$$
\begin{aligned}
& \|u\|_{W^{2, p}\left(D_{3 / 4}^{+}\right)}+\|v\|_{W^{2, p}\left(D_{3 / 4}^{+}\right)} \leq\|\eta u\|_{W^{2, p}\left(D^{+}\right)}+\|\eta v\|_{W^{2, p}\left(D^{+}\right)} \\
& \quad \leq C\left(\|(\nabla u, \nabla v)\|_{L^{2}\left(D^{+}\right)}+\||(\nabla \phi, \nabla \psi)|\|_{W^{1, p}\left(D^{+}\right)}+\||(\tau, \kappa)|\|_{L^{p}\left(D^{+}\right)}\right) .
\end{aligned}
$$

Thus we have proved the lemma for the case $1<p<2$.

If $p=2$, one can derive the above estimate for $p=\frac{4}{3}$ at first. Such an estimate implies that $\nabla u$ and $\nabla v$ are bounded in $L^{4}\left(D_{3 / 4}^{+}\right)$. Then one can apply the $W^{2,2}$-boundary estimate to the equation and get the conclusion of the lemma with $p=2$. 
For an approximate Lorentzian harmonic map, we can prove the following Pohozaev type identity which is useful in the blow-up analysis. This kind of equality was first introduced in [20] for the interior case of harmonic maps and extended in $[10,15,16]$ for some boundary cases.

Lemma 2.3 Let $D \subset \mathbb{R}^{2}$ be the unit disk and $(u, v) \in W^{2,2}(D, N \times \mathbb{R})$ be an approximate Lorentzian harmonic map with $(\tau, \kappa) \in L^{2}(D)$, then for any $0<\rho<\frac{1}{2}$, we have

$$
\begin{aligned}
& \rho \int_{\partial D_{\rho}}\left(\left|u_{r}\right|^{2}-\beta(u)\left|v_{r}+\omega_{i} u_{r}^{i}\right|^{2}-\frac{1}{2}|\nabla u|^{2}+\frac{1}{2} \beta(u)\left|\nabla v+\omega_{i} \nabla u^{i}\right|^{2}\right) d s \\
& =\int_{D_{\rho}} r u_{r} \tau d x-\int_{D_{\rho}} r\left(v_{r}+\omega_{i} u_{r}^{i}\right) \kappa d x .
\end{aligned}
$$

where $(r, \theta)$ are polar coordinates in $D$ centered at 0 . Since we use the Euclidean metric, we have that the covariant derivative $\nabla_{r} u$ equals to $\frac{\partial u}{\partial r}$ and we denote them with a unified notation $\frac{\partial u}{\partial r}$ or just $u_{r}$ for brevity.

Proof Multiplying (1.8) by $r\left(v_{r}+\omega_{i} u_{r}^{i}\right)$ and integrating by parts, we get

$$
\begin{aligned}
\int_{D_{\rho}} & r\left(v_{r}+\omega_{i} u_{r}^{i}\right) \kappa d x \\
= & \int_{D_{\rho}} \operatorname{div}\left(\beta(u)\left(\nabla v+\omega_{i} \nabla u^{i}\right)\right) \cdot r\left(v_{r}+\omega_{i} u_{r}^{i}\right) d x \\
= & \int_{\partial D_{\rho}} r \beta(u)\left|v_{r}+\omega_{i} u_{r}^{i}\right|^{2} d s-\int_{D_{\rho}} \beta(u)\left(\nabla v+\omega_{i} \nabla u^{i}\right) \cdot \nabla\left(r\left(v_{r}+\omega_{i} u_{r}^{i}\right)\right) d x \\
= & \int_{\partial D_{\rho}} r \beta(u)\left|v_{r}+\omega_{i} u_{r}^{i}\right|^{2} d s-\int_{D_{\rho}} \beta(u)\left|\nabla v+\omega_{i} \nabla u^{i}\right|^{2} d x \\
& -\int_{D_{\rho}} \beta(u)\left(\nabla v+\omega_{i} \nabla u^{i}\right) \cdot r\left(\frac{\partial}{\partial r}\left(\nabla v+\omega_{i} \nabla u^{i}\right)-\frac{\partial \omega_{i}}{\partial r} \nabla u^{i}+\frac{\partial u_{i}}{\partial r} \nabla \omega^{i}\right) d x .
\end{aligned}
$$

By direct computations, noting that

$$
\begin{aligned}
& -\int_{D_{\rho}} \beta(u)\left(\nabla v+\omega_{i} \nabla u^{i}\right) \cdot r \frac{\partial}{\partial r}\left(\nabla v+\omega_{i} \nabla u^{i}\right) d x \\
& =-\frac{1}{2} \int_{D_{\rho}} \beta(u) r \frac{\partial}{\partial r}\left|\nabla v+\omega_{i} \nabla u^{i}\right|^{2} d x \\
& =-\frac{1}{2} \int_{\partial D_{\rho}} r \beta(u)\left|\nabla v+\omega_{i} \nabla u^{i}\right|^{2} d s+\int_{D_{\rho}} \beta(u)\left|\nabla v+\omega_{i} \nabla u^{i}\right|^{2} d x \\
& \quad+\frac{1}{2} \int_{D_{\rho}} r \frac{\partial \beta(u)}{\partial r}\left|\nabla v+\omega_{i} \nabla u^{i}\right|^{2} d x,
\end{aligned}
$$

we have

$$
\begin{aligned}
\int_{D_{\rho}} r\left(v_{r}+\omega_{i} u_{r}^{i}\right) \kappa d x= & \int_{\partial D_{\rho}} r \beta(u)\left(\left|v_{r}+\omega_{i} u_{r}^{i}\right|^{2}-\frac{1}{2}\left|\nabla v+\omega_{i} \nabla u^{i}\right|^{2}\right) d s \\
& +\int_{D_{\rho}} \beta(u)\left(\nabla v+\omega_{i} \nabla u^{i}\right) \cdot r\left(\frac{\partial \omega_{i}}{\partial r} \nabla u^{i}-\frac{\partial u_{i}}{\partial r} \nabla \omega^{i}\right) d x \\
& +\frac{1}{2} \int_{D_{\rho}} r \frac{\partial \beta(u)}{\partial r}\left|\nabla v+\omega_{i} \nabla u^{i}\right|^{2} d x
\end{aligned}
$$


Similarly, multiplying (1.7) by $r u_{r}$ and integrating by parts, we get

$$
\begin{aligned}
\int_{D_{\rho}} r u_{r} \tau d x & =\int_{D_{\rho}}\left(\Delta u-H^{\top}\right) r u_{r} d x \\
& =\int_{\partial D_{\rho}} r\left|u_{r}\right|^{2} d s-\int_{D_{\rho}} \nabla u \cdot \nabla\left(r u_{r}\right) d x-\int_{D_{\rho}} H \cdot r u_{r} d x \\
& =\int_{\partial D_{\rho}} r\left(\left|u_{r}\right|^{2}-\frac{1}{2}|\nabla u|^{2}\right)-\int_{D_{\rho}} H \cdot r u_{r} d x
\end{aligned}
$$

Noting that

$$
\begin{aligned}
H \cdot r u_{r} & =r H^{j} u_{r}^{j} \\
& =r\left(\beta\left(\nabla v+\omega_{i} \nabla u^{i}\right) \cdot \nabla u^{k}\left(\frac{\partial \omega_{j}}{\partial y^{k}}-\frac{\partial \omega_{k}}{\partial y^{j}}\right)-\frac{1}{2} \frac{\partial \beta}{\partial y^{j}}\left|\nabla v+\omega_{i} \nabla u^{i}\right|^{2}\right) u_{r}^{j} \\
& =r \beta\left(\nabla v+\omega_{i} \nabla u^{i}\right)\left(u_{r}^{j} \nabla \omega_{j}-\frac{\partial \omega_{j}}{\partial r} \nabla u^{j}\right)-\frac{1}{2} r \frac{\partial \beta(u)}{\partial r}\left|\nabla v+\omega_{i} \nabla u^{i}\right|^{2}
\end{aligned}
$$

and combining (2.4) with (2.2) and (2.3), we obtain the conclusion of the lemma.

By Hölder's inequality and integrating (2.1) about $\rho$ from $r_{0}$ to $2 r_{0}$, we get

Corollary 2.4 For $(u, v)$ in Lemma 2.3, if $\|(\nabla u, \nabla v)\|_{L^{2}(D)}+\|(\tau, \kappa)\|_{L^{2}(D)} \leq \Lambda$, then for any $0<r_{0}<\frac{1}{4}$, we have

$$
\int_{D_{2 r_{0}} \backslash D_{r_{0}}}\left(\left|u_{r}\right|^{2}-\beta(u)\left|v_{r}+\omega_{i} u_{r}^{i}\right|^{2}-\frac{1}{2}|\nabla u|^{2}+\frac{1}{2} \beta(u)\left|\nabla v+\omega_{i} \nabla u^{i}\right|^{2}\right) d x \leq C r_{0},
$$

where $C=C\left(\lambda_{1}, \lambda_{2}, \Lambda, N\right)>0$ is a constant.

Denote $\tilde{u}=u-\phi$ and $\tilde{v}=v-\psi$. For the boundary case, we have

Lemma 2.5 Let $D^{+} \subset \mathbb{R}^{2}$ be the upper unit disk and $(u, v) \in W^{2,2}\left(D^{+}, N \times \mathbb{R}\right)$ be an approximate Lorentzian harmonic map with Dirichlet boundary data $\left.(u, v)\right|_{\partial^{0} D^{+}}=(\phi, \psi) \in$ $C^{2+\alpha}(D)$ and $(\tau, \kappa) \in L^{2}\left(D^{+}\right)$, then for any $0<\rho<\frac{1}{2}$, we have

$$
\begin{aligned}
& \rho \int_{\partial^{+} D_{\rho}^{+}}\left(\left|u_{r}\right|^{2}-\beta(u)\left|v_{r}+\omega_{i} u_{r}^{i}\right|^{2}-\frac{1}{2}|\nabla u|^{2}+\frac{1}{2} \beta(u)\left|\nabla v+\omega_{i} \nabla u^{i}\right|^{2}\right) d s \\
& =\int_{D_{\rho}^{+}} r \widetilde{u}_{r} \tau d x-\int_{D_{\rho}^{+}} r\left(\widetilde{v}_{r}+\omega_{i} \widetilde{u}_{r}^{i}\right) \kappa d x+\int_{\partial^{+} D_{\rho}^{+}} r \frac{\partial u}{\partial r} \frac{\partial \phi}{\partial r} d s-\int_{D_{\rho}^{+}} \nabla u \nabla\left(r \phi_{r}\right) d x \\
& \quad+\int_{D_{\rho}^{+}}\left(A(u)(\nabla u, \nabla u)-H^{\top}\right) \cdot r \phi_{r} d x-\int_{\partial^{+} D_{\rho}^{+}} r \beta(u)\left(v_{r}+\omega_{i} u_{r}^{i}\right)\left(\psi_{r}+\omega_{i} \phi_{r}^{i}\right) d s \\
& \quad+\int_{D_{\rho}^{+}} \beta(u)\left(\nabla v+\omega_{i} \nabla u^{i}\right) \cdot\left(\nabla \psi+\omega_{i} \nabla \phi^{i}\right) d x \\
& \quad+\int_{D_{\rho}^{+}} r \beta(u)\left(\nabla v+\omega_{i} \nabla u^{i}\right) \cdot \nabla\left(\psi_{r}+\omega_{i} \phi_{r}^{i}\right) d x
\end{aligned}
$$

where $(r, \theta)$ are polar coordinates in $D$ centered at 0 .

Proof The proof is similar to the proof of Lemma 2.3. 
Multiplying (1.8) by $r\left(\widetilde{v}_{r}+\omega_{i} \widetilde{u}_{r}^{i}\right)$ and integrating by parts, we get

$$
\begin{aligned}
\int_{D_{\rho}^{+}} r\left(\widetilde{v}_{r}+\omega_{i} \widetilde{u}_{r}^{i}\right) \kappa d x= & \int_{D_{\rho}^{+}} \operatorname{div}\left(\beta(u)\left(\nabla v+\omega_{i} \nabla u^{i}\right)\right) \cdot r\left(\widetilde{v}_{r}+\omega_{i} \widetilde{u}_{r}^{i}\right) d x \\
= & \int_{\partial^{+} D_{\rho}^{+}} \beta(u)\left(v_{r}+\omega_{i} u_{r}^{i}\right) \cdot r\left(\widetilde{v}_{r}+\omega_{i} \widetilde{u}_{r}^{i}\right) d s \\
& -\int_{D_{\rho}^{+}} \beta(u)\left(\nabla v+\omega_{i} \nabla u^{i}\right) \cdot \nabla\left(r\left(\widetilde{v}_{r}+\omega_{i} \widetilde{u}_{r}^{i}\right)\right) d x \\
= & \int_{\partial^{+} D_{\rho}^{+}} \beta(u)\left(v_{r}+\omega_{i} u_{r}^{i}\right) \cdot r\left(\widetilde{v}_{r}+\omega_{i} \widetilde{u}_{r}^{i}\right) d s \\
& -\int_{D_{\rho}^{+}} \beta(u)\left(\nabla v+\omega_{i} \nabla u^{i}\right) \cdot\left(\nabla \widetilde{v}+\omega_{i} \nabla \widetilde{u}^{i}\right) d x \\
& -\int_{D_{\rho}^{+}} r \beta(u)\left(\nabla v+\omega_{i} \nabla u^{i}\right) \cdot \nabla\left(\widetilde{v}_{r}+\omega_{i} \widetilde{u}_{r}^{i}\right) d x=\mathbb{I}+\mathbb{I I I}+\mathbb{I I I I} .
\end{aligned}
$$

By direct computations, we have

$$
\begin{aligned}
\mathbb{I} & =\int_{\partial^{+} D_{\rho}^{+}} r \beta(u)\left|v_{r}+\omega_{i} u_{r}^{i}\right|^{2} d s-\int_{\partial^{+} D_{\rho}^{+}} r \beta(u)\left(v_{r}+\omega_{i} u_{r}^{i}\right)\left(\psi_{r}+\omega_{i} \phi_{r}^{i}\right) d s, \\
\mathbb{I I} & =-\int_{D_{\rho}^{+}} \beta(u)\left|\nabla v+\omega_{i} \nabla u^{i}\right|^{2} d x+\int_{D_{\rho}^{+}} \beta(u)\left(\nabla v+\omega_{i} \nabla u^{i}\right) \cdot\left(\nabla \psi+\omega_{i} \nabla \phi^{i}\right) d x,
\end{aligned}
$$

and

$$
\begin{aligned}
\mathbb{I I I I}= & -\frac{1}{2} \int_{D_{\rho}^{+}} r \beta(u) \frac{\partial}{\partial r}\left|\nabla v+\omega_{i} \nabla u^{i}\right|^{2} d x+\int_{D_{\rho}^{+}} r \beta(u)\left(\nabla v+\omega_{i} \nabla u^{i}\right) \cdot \nabla\left(\psi_{r}+\omega_{i} \phi_{r}^{i}\right) d x \\
& +\int_{D_{\rho}^{+}} r \beta(u)\left(\nabla v+\omega_{i} \nabla u^{i}\right) \cdot\left(\frac{\partial \omega_{i}}{\partial r} \nabla u^{i}-\nabla \omega_{i} u_{r}^{i}\right) d x .
\end{aligned}
$$

Noting that

$$
\begin{aligned}
- & \frac{1}{2} \int_{D_{\rho}^{+}} \beta(u) r \frac{\partial}{\partial r}\left|\nabla v+\omega_{i} \nabla u^{i}\right|^{2} d x \\
= & -\frac{1}{2} \int_{\partial^{+} D_{\rho}^{+}} r \beta(u)\left|\nabla v+\omega_{i} \nabla u^{i}\right|^{2} d s+\int_{D_{\rho}^{+}} \beta(u)\left|\nabla v+\omega_{i} \nabla u^{i}\right|^{2} d x \\
& +\frac{1}{2} \int_{D_{\rho}^{+}} r \frac{\partial \beta(u)}{\partial r}\left|\nabla v+\omega_{i} \nabla u^{i}\right|^{2} d x,
\end{aligned}
$$


we have

$$
\begin{aligned}
\int_{D_{\rho}^{+}} r\left(\widetilde{v}_{r}+\omega_{i} \widetilde{u}_{r}^{i}\right) \kappa d x= & \int_{\partial^{+} D_{\rho}^{+}} r \beta(u)\left(\left|v_{r}+\omega_{i} u_{r}^{i}\right|^{2}-\frac{1}{2}\left|\nabla v+\omega_{i} \nabla u^{i}\right|^{2}\right) d s \\
& +\int_{D_{\rho}^{+}} \beta(u)\left(\nabla v+\omega_{i} \nabla u^{i}\right) \cdot r\left(\frac{\partial \omega_{i}}{\partial r} \nabla u^{i}-\frac{\partial u_{i}}{\partial r} \nabla \omega^{i}\right) d x \\
& +\frac{1}{2} \int_{D_{\rho}^{+}} r \frac{\partial \beta(u)}{\partial r}\left|\nabla v+\omega_{i} \nabla u^{i}\right|^{2} d x \\
& -\int_{\partial^{+} D_{\rho}^{+}} r \beta(u)\left(v_{r}+\omega_{i} u_{r}^{i}\right)\left(\psi_{r}+\omega_{i} \phi_{r}^{i}\right) d s \\
& +\int_{D_{\rho}^{+}} \beta(u)\left(\nabla v+\omega_{i} \nabla u^{i}\right) \cdot\left(\nabla \psi+\omega_{i} \nabla \phi^{i}\right) d x \\
& +\int_{D_{\rho}^{+}} r \beta(u)\left(\nabla v+\omega_{i} \nabla u^{i}\right) \cdot \nabla\left(\psi_{r}+\omega_{i} \phi_{r}^{i}\right) d x .
\end{aligned}
$$

Similarly, multiplying (1.7) by $r \tilde{u}_{r}$ and integrating by parts, we get

$$
\begin{aligned}
\int_{D_{\rho}^{+}} r \tilde{u}_{r} \tau d x= & \int_{D_{\rho}^{+}}\left(\Delta u+A(u)(\nabla u, \nabla u)-H^{\top}\right) \cdot r \tilde{u}_{r} d x \\
= & \int_{\partial^{+} D_{\rho}^{+}} r\left(\left|u_{r}\right|^{2}-\frac{1}{2}|\nabla u|^{2}\right) d s-\int_{\partial^{+} D_{\rho}^{+}} r \frac{\partial u}{\partial r} \frac{\partial \phi}{\partial r}+\int_{D_{\rho}^{+}} \nabla u \nabla\left(r \phi_{r}\right) d x \\
& -\int_{D_{\rho}^{+}}\left(A(u)(\nabla u, \nabla u)-H^{\top}\right) \cdot r \phi_{r} d x-\int_{D_{\rho}^{+}} H \cdot r u_{r} d x
\end{aligned}
$$

Combining (2.4) with (2.6) and (2.7), we obtain the conclusion of the lemma.

Corollary 2.6 For $(u, v)$ in Lemma 2.5 , if $\|(\nabla u, \nabla v)\|_{L^{2}\left(D^{+}\right)}+\|(\tau, \kappa)\|_{L^{2}\left(D^{+}\right)} \leq \Lambda$, then for any $0<r_{0}<\frac{1}{4}$, we have

$$
\int_{D_{2 r_{0}}^{+} \backslash D_{r_{0}}^{+}}\left(\left|u_{r}\right|^{2}-\beta(u)\left|v_{r}+\omega_{i} u_{r}^{i}\right|^{2}-\frac{1}{2}|\nabla u|^{2}+\frac{1}{2} \beta(u)\left|\nabla v+\omega_{i} \nabla u^{i}\right|^{2}\right) d x \leq C r_{0},
$$

where $C=C\left(\lambda_{1}, \lambda_{2}, \Lambda, N\right)>0$ is a constant.

Proof By Hölder's inequality, it is easy to find that the right hand side of (2.5) is bounded by

$$
C \rho \int_{\partial^{+} D_{\rho}^{+}}(|\nabla u|+|\nabla v|)+C \rho,
$$

where $C=C\left(\lambda_{1}, \lambda_{2}, N,\|\phi\|_{C^{2}},\|\psi\|_{C^{2}}\right)$. Then the conclusion of the corollary follows by an integration about $\rho$ from $r_{0}$ to $2 r_{0}$.

Similar as for harmonic maps into a Riemannian manifold, there is also an energy gap for a nontrivial Lorentzian harmonic map.

Theorem 2.7 (Gap phenomenon) Suppose either $(u, v): \mathbb{S}^{2} \rightarrow N \times \mathbb{R}$ is a smooth Lorentzian harmonic map or $(u, v): \mathbb{R}_{+}^{2} \rightarrow N \times \mathbb{R}$ is a smooth Lorentzian harmonic map with Dirichlet boundary condition:

$$
\left.(u, v)\right|_{\partial \mathbb{R}_{+}^{2}}=\text { constant }
$$


then there exists a constant $\epsilon_{0}>0$ depending on $(N \times \mathbb{R}, g)$, such that if either

$$
E\left(u, v ; \mathbb{S}^{2}\right) \leq \epsilon_{0} \text { or } E\left(u, v ; \mathbb{R}_{+}^{2}\right) \leq \epsilon_{0},
$$

then $(u, v)$ is a constant map. Here $\mathbb{S}^{2}$ denotes the unit sphere in $\mathbb{R}^{3}$.

Proof One can find the proof of the theorem in [9] for the case $(u, v): \mathbb{S}^{2} \rightarrow N \times \mathbb{R}$.

By Eqs. (1.4) and (1.5), we have

$$
|\Delta u|+|\Delta v| \leq C\left(|\nabla u|^{2}+|\nabla v|^{2}\right) .
$$

The standard elliptic theory tells us that

$$
\begin{aligned}
\|\nabla u\|_{W^{1, \frac{4}{3}}}+\|\nabla v\|_{W^{1, \frac{4}{3}}} & \leq C\left(\|\Delta u\|_{L^{\frac{4}{3}}}+\|\Delta v\|_{L^{\frac{4}{3}}}\right) \\
& \leq C E^{\frac{1}{2}}\left(u, v ; \mathbb{S}^{2}\right)\left(\|\nabla u\|_{L^{4}}+\|\nabla v\|_{L^{4}}\right) \\
& \leq C \sqrt{\epsilon_{0}}\left(\|\nabla u\|_{W^{1, \frac{4}{3}}}+\|\nabla v\|_{W^{1, \frac{4}{3}}}\right) .
\end{aligned}
$$

It is easy to get that, if $\epsilon_{0}$ is small enough, $(u, v)$ must be a constant map.

If $(u, v): \mathbb{R}_{+}^{2} \rightarrow N \times \mathbb{R}$ is a smooth Lorentzian harmonic map with constant Dirichlet boundary condition, choosing $\epsilon_{0} \leq \epsilon_{2}$ where $\epsilon_{2}$ is the positive constant in Lemma 2.2, then by Lemma 2.2 (taking $(\phi, \psi)=$ constant,$(\tau, \kappa)=0$ and any constant $p>2$ ) and Sobolev embedding, for any $R>0$, we have

$$
R\|\nabla u\|_{L^{\infty}\left(D_{R / 2}^{+}\right)}+R\|\nabla v\|_{L^{\infty}\left(D_{R / 2}^{+}\right)} \leq C E^{\frac{1}{2}}\left(u, v ; D_{R}^{+}\right) \leq C \epsilon_{0}^{\frac{1}{2}} .
$$

Sending $R$ to infinity yields that $(u, v)$ must be a constant map.

It is necessary for the singularities to be removable during the blow-up process. Removability of singularities for a Lorentzian harmonic map (i.e. $\tau=\kappa=0$ ) is proved in [9]. By assuming additionally that $\omega \equiv 0$, for an approximate Lorentzian harmonic map (i.e. $(\tau, \kappa) \neq 0$ ) with singularities either in the interior or on the boundary, we can also remove them.

Theorem 2.8 Suppose $(u, v) \in W_{l o c}^{2,2}(D \backslash\{0\})$ is an approximate Lorentzian harmonic map from the punctured disk $D \backslash\{0\}$ to $\left(N \times \mathbb{R}, g_{N}-\beta d^{2} \theta\right)$. If $E(u, v ; D)<\infty$ and $(\tau, \kappa) \in L^{2}(D)$, then $(u, v)$ can be extended to the whole disk $D$ in $W^{2,2}(D)$.

For an approximate Lorentzian harmonic map $(u, v) \in W_{l o c}^{2,2}\left(D^{+} \backslash\{0\}\right)$ which is from $D^{+} \backslash\{0\}$ to $\left(N \times \mathbb{R}, g_{N}-\beta d^{2} \theta\right)$ with boundary data $\left.(u, v)\right|_{\partial^{0} D^{+}}=(\phi, \psi)$. If $E\left(u, v ; D^{+}\right)<$ $\infty$ and $(\tau, \kappa) \in L^{2}\left(D^{+}\right)$, then $(u, v)$ can also be extended to $D^{+}$in $W^{2,2}\left(D^{+}\right)$.

Proof We prove the theorem for the boundary case and the interior case can be proved similarly.

On the one hand, it is easy to see that $(u, v)$ is a weak solution of (1.7) and (1.8). By Theorem 1.2 in [30] which is developed from the regularity theory for critical elliptic systems with an anti-symmetric structure in $[25,26,28,29,32]$, we know that $v \in W^{2, p}\left(D_{\rho}^{+}(0)\right)$ for some $\rho>0$ and any $1<p<2$. In fact, the anti-symmetric term in the equation for $v$ equals to zero. This implies that $\nabla v \in L^{4}\left(D^{+}\right)$.

On the other hand, since the Eq. (1.7) can be written as an elliptic system with an antisymmetric potential ([25])

$$
\Delta u=\Omega \cdot \nabla u+f
$$


with $\Omega \in L^{2}\left(D^{+}, s o(n) \otimes \mathbb{R}^{2}\right)$ and $f \in L^{2}\left(D^{+}\right)$, using Theorem 1.2 in [30] again, we have $u \in W^{2, p}\left(D_{\rho}^{+}(0)\right)$ for some $\rho>0$ and any $1<p<2$. Then the higher regularity can be derived by a standard bootstrap argument.

\section{Energy identity and analysis on the neck}

In this section, we shall study the behavior at blow-up points both in the interior and on the boundary for an approximate Lorentzian harmonic map sequence $\left\{\left(u_{n}, v_{n}\right)\right\}$. To this end, we first define the blow-up set and show that the blow-up points for such a sequence are finite in number. Throughout this section, we suppose that there exists a constant $\Lambda>0$ such that the sequence satisfies

$$
\left\|\left(\nabla u_{n}, \nabla v_{n}\right)\right\|_{L^{2}\left(D_{1}(0)\right)}+\left\|\left(\tau_{n}, \kappa_{n}\right)\right\|_{L^{2}\left(D_{1}(0)\right)} \leq \Lambda .
$$

Definition 3.1 For an approximate Lorentzian harmonic map sequence $\left\{\left(u_{n}, v_{n}\right)\right\}$, define

$$
\mathcal{S}_{1}:=\cap_{r>0}\left\{x \in M \mid \liminf _{n \rightarrow \infty} \int_{D_{r}(x)}\left(\left|\nabla u_{n}\right|^{2}+\left|\nabla v_{n}\right|^{2}\right) d v_{h} \geq \epsilon_{1}\right\},
$$

and

$$
\mathcal{S}_{2}:=\cap_{r>0}\left\{x \in \partial M \mid \liminf _{n \rightarrow \infty} \int_{D_{r}^{+}(x)}\left(\left|\nabla u_{n}\right|^{2}+\left|\nabla v_{n}\right|^{2}\right) d v_{h} \geq \epsilon_{2}\right\},
$$

where $\epsilon_{1}$ and $\epsilon_{2}$ are constants in Lemmas 2.1 and 2.2. The blow-up set of $\left\{\left(u_{n}, v_{n}\right)\right\}$ is defined to be $\mathcal{S}:=\mathcal{S}_{1} \cup \mathcal{S}_{2}$.

Lemma 3.1 For an approximate harmonic map sequence satisfying (3.1), the blow-up set $\mathcal{S}$ is a finite set.

Proof By (3.1), we can take a subsequence and still denote it by $\left\{\left(u_{n}, v_{n}\right)\right\}$, such that $\left\{\left(u_{n}, v_{n}\right)\right\}$ converges weakly in $W^{1,2}(M)$ to a limit map $(u, v): M \rightarrow(N \times \mathbb{R}, g)$. If for any point $x \in M$,

$$
\lim _{r \rightarrow 0} \limsup _{n \rightarrow \infty} \int_{D_{r}(x)}\left|\nabla u_{n}\right|^{2}+\left|\nabla v_{n}\right|^{2}<\epsilon_{1},
$$

Lemma 2.1 tells that the convergence is strong in $W^{1,2}(M)$. Obviously in this case, $\mathcal{S}_{1}$ is empty. Otherwise, if there exists a point $p_{1} \in M$ such that

$$
\lim _{r \rightarrow 0} \limsup _{n \rightarrow \infty} \int_{D_{r}\left(p_{1}\right)}\left|\nabla u_{n}\right|^{2}+\left|\nabla v_{n}\right|^{2} \geq \epsilon_{1},
$$

By taking a subsequence, we can assume that

$$
\lim _{r \rightarrow 0} \lim _{n \rightarrow \infty} \int_{D_{r}\left(p_{1}\right)}\left|\nabla u_{n}\right|^{2}+\left|\nabla v_{n}\right|^{2} \geq \epsilon_{1}
$$

If (3.2) holds for any point $x \in M \backslash\left\{p_{1}\right\}$, we get that $\mathcal{S}_{1}=\left\{p_{1}\right\}$. Otherwise, we can find a point $p_{2}$ where the energy concentration (3.3) happens. Since the energy of the sequence is bounded, this process must stop after finite steps.

For points on the boundary of $M$, we can proceed similarly and finally, we get a subsequence $\left\{\left(u_{n}, v_{n}\right)\right\}$ which converges strongly to some $(u, v)$ in $W_{l o c}^{1,2}(M \backslash \mathcal{S})$, where $\mathcal{S}=\mathcal{S}_{1} \cup \mathcal{S}_{2}=\left\{p_{1}, p_{2}, \ldots, p_{I}\right\}$ is a finite set. 
We consider the case that the blow-up points are in the interior first. Since the blow-up set $\mathcal{S}_{1}$ is finite, we can find small geodesic disks $D_{\delta_{i}}$ (by conformal invariance, we can assume that they are flat disks) for each blow-up point $p_{i}$ such that $D_{\delta_{i}} \cap D_{\delta_{j}}=\varnothing$ for $i \neq j$, $i, j=1,2, \ldots, I$, and on $M \backslash \cup_{i=1}^{I} D_{\delta_{i}},\left(u_{n}, v_{n}\right)$ converges strongly to a limit map $(u, v)$. Without loss of generality, we discuss the case that there is only one blow-up point $0 \in D_{1}(0)$ in $\mathcal{S}_{1}$ and the sequence $\left\{\left(u_{n}, v_{n}\right)\right\}$ satisfies that there is some $(u, v)$ such that

$$
\left(u_{n}, v_{n}\right) \rightarrow(u, v) \text { weakly in } W_{l o c}^{2,2}\left(D_{1}(0) \backslash\{0\}\right) \text { as } n \rightarrow \infty .
$$

Lemma 3.2 Let $\left\{\left(u_{n}, v_{n}\right)\right\} \in W^{2,2}\left(D_{1}(0), N \times \mathbb{R}\right)$ be a sequence of approximate Lorentzian harmonic maps satisfying (3.1) and (3.4). Up to a subsequence which is still denoted by $\left\{\left(u_{n}, v_{n}\right)\right\}$, there exist a positive integer $L$ and nontrivial Lorentzian harmonic spheres $\left(\sigma^{i}, \xi^{i}\right): \mathbb{R}^{2} \cup\{\infty\} \rightarrow N \times \mathbb{R}, i=1, \ldots$, L satisfying

$$
\lim _{n \rightarrow \infty} E_{g}\left(u_{n}, v_{n} ; D_{1}(0)\right)=E_{g}\left(u, v ; D_{1}(0)\right)+\sum_{i=1}^{L} E_{g}\left(\sigma^{i}, \xi^{i}\right) .
$$

Proof According to the standard induction argument in [5], we can assume that there is only one bubble at the singular point $0 \in D_{1}(0)$. To prove (3.5) is equivalent to prove that there exists a Lorentzian harmonic sphere $(\sigma, \xi)$ such that

$$
\lim _{\delta \rightarrow 0} \lim _{n \rightarrow \infty} E_{g}\left(u_{n}, v_{n}, D_{\delta}\right)=E_{g}(\sigma, \xi) .
$$

By the standard argument of blow-up analysis, for any $n$, there exist sequences $x_{n} \rightarrow 0$ and $r_{n} \rightarrow 0$ such that

$$
E\left(u_{n}, v_{n} ; D_{r_{n} / 2}\left(x_{n}\right)\right)=\sup _{\substack{x \in D_{\delta}, r \leq r_{n} \\ D_{r}(x) \subset D_{\delta}}} E\left(u_{n}, v_{n} ; D_{r / 2}(x)\right)=\frac{\epsilon_{1}}{8} .
$$

Without loss of generality, we may assume that $x_{n}=0$ and denote $\tilde{u}_{n}=u_{n}\left(r_{n} x\right), \quad \tilde{v}_{n}=$ $v_{n}\left(r_{n} x\right)$. Then we have

$$
E\left(\tilde{u}_{n}, \tilde{v}_{n} ; D_{1 / 2}\right)=E\left(u_{n}, v_{n} ; D_{r_{n} / 2}\right)=\frac{\epsilon_{1}}{8}<\epsilon_{1}
$$

and

$$
E\left(\tilde{u}_{n}, \tilde{v}_{n} ; D_{R}\right)=E\left(u_{n}, v_{n} ; D_{r_{n} R}\right)<\Lambda .
$$

By (3.7), we can apply Lemma 2.1 on $D_{R}$ for $\left\{\left(\tilde{u}_{n}, \tilde{v}_{n}\right)\right\}$ and get that $\left\{\left(\tilde{u}_{n}, \tilde{v}_{n}\right)\right\}$ converges strongly to some Lorentzian harmonic map $(\sigma, \xi)$ in $W^{1,2}\left(D_{R}, N \times \mathbb{R}\right)$ for any $R \geq 1$. By stereographic projection and the removable singularity theorem [9], we get a nonconstant harmonic sphere $(\sigma, \xi)$. Thus we get the first bubble at the blow-up point and to prove (3.6) is equivalent to prove that

$$
\lim _{R \rightarrow \infty} \lim _{\delta \rightarrow 0} \lim _{n \rightarrow \infty} E_{g}\left(u_{n}, v_{n} ; D_{\delta}(0) \backslash D_{r_{n} R}(0)\right)=0 .
$$

Since we assume that there is only one bubble, we have that, for any $\epsilon>0$, there holds that

$$
E\left(u_{n}, v_{n} ; D_{4 \rho} \backslash D_{\frac{\rho}{2}}\right) \leq \epsilon^{2} \text { for } \rho \in\left[r_{n} R, \frac{\delta}{2}\right]
$$

as $n \rightarrow \infty, R \rightarrow \infty$ and $\delta \rightarrow 0$. Otherwise, we will get a second bubble and this is a contradiction to the assumption that $L=1$. One can refer to $[5,15,31]$ for details of this kind 
of arguments. Then by Lemma 2.1 and a standard scaling argument, for any $\rho \in\left[r_{n} R, \frac{\delta}{2}\right]$, we have

$$
\begin{aligned}
& \left\|u_{n}\right\|_{\operatorname{osc}\left(D_{2 \rho} \backslash D_{\rho}\right)}+\left\|v_{n}\right\|_{o s c}\left(D_{2 \rho} \backslash D_{\rho}\right) \\
& \quad \leq C E^{\frac{1}{2}}\left(u_{n}, v_{n} ; D_{4 \rho} \backslash D_{\frac{\rho}{2}}\right)+C \rho\left\|\left(\tau_{n}, \kappa_{n}\right)\right\|_{L^{2}\left(D_{4 \rho} \backslash D_{\frac{\rho}{2}}\right)} .
\end{aligned}
$$

Define

$$
u_{n}^{*}(r):=\frac{1}{2 \pi} \int_{0}^{2 \pi} u_{n}(r, \theta) d \theta, v_{n}^{*}(r):=\frac{1}{2 \pi} \int_{0}^{2 \pi} v_{n}(r, \theta) d \theta .
$$

By (3.11), we know that

$$
\begin{aligned}
\left\|u_{n}-u_{n}^{*}\right\|_{L^{\infty}\left(D_{\delta} \backslash D_{r_{n} R}\right)} & =\sup _{r_{n} R \leq t \leq \frac{\delta}{2}}\left\|u_{n}-u_{n}^{*}\right\|_{L^{\infty}\left(D_{2 t} \backslash D_{t}\right)} \\
& \leq \sup _{r_{n} R \leq t \leq \frac{\delta}{2}}\left\|u_{n}\right\|_{\operatorname{osc}\left(D_{2 t} \backslash D_{t}\right) \leq C(\epsilon+\delta)} \leq C
\end{aligned}
$$

and similarly,

$$
\left\|v_{n}-v_{n}^{*}\right\|_{L^{\infty}\left(D_{\delta} \backslash D_{r_{n} R}\right)} \leq C(\epsilon+\delta) .
$$

Then we get by integrating by parts that

$$
\begin{aligned}
& \int_{D_{\delta} \backslash D_{r_{n} R}}-\Delta u_{n}\left(u_{n}-u_{n}^{*}\right) d x \\
& =\int_{D_{\delta} \backslash D_{r_{n} R}} \nabla u_{n} \nabla\left(u_{n}-u_{n}^{*}\right) d x-\int_{\partial D_{\delta}} \frac{\partial u_{n}}{\partial r}\left(u_{n}-u_{n}^{*}\right)+\int_{\partial D_{r_{n} R}} \frac{\partial u_{n}}{\partial r}\left(u_{n}-u_{n}^{*}\right) \\
& \geq \int_{D_{\delta} \backslash D_{r_{n} R}}\left|\nabla u_{n}\right|^{2} d x-\int_{D_{\delta} \backslash D_{r_{n} R}}\left|\frac{\partial u_{n}}{\partial r}\right|^{2} d x-\int_{\partial D_{\delta}} \frac{\partial u_{n}}{\partial r}\left(u_{n}-u_{n}^{*}\right) \\
& +\int_{\partial D_{r_{n} R}} \frac{\partial u_{n}}{\partial r}\left(u_{n}-u_{n}^{*}\right) \\
& =\int_{D_{\delta} \backslash D_{r_{n} R}}\left|r^{-1} \frac{\partial u_{n}}{\partial \theta}\right|^{2} d x-\int_{\partial D_{\delta}} \frac{\partial u_{n}}{\partial r}\left(u_{n}-u_{n}^{*}\right)+\int_{\partial D_{r_{n} R} R} \frac{\partial u_{n}}{\partial r}\left(u_{n}-u_{n}^{*}\right) .
\end{aligned}
$$

Since $\left(u_{n}, v_{n}\right)$ is an approximate harmonic map, we have

$$
\left|\Delta u_{n}\right|+\left|\Delta v_{n}\right| \leq C\left(\lambda_{1}, \lambda_{2}, N\right)\left(\left|\nabla u_{n}\right|^{2}+\left|\nabla v_{n}\right|^{2}\right) .
$$

Then we get from (3.12), (3.13) and (3.14) that

$$
\begin{aligned}
& \int_{D_{\delta} \backslash D_{r_{n} R}}\left|r^{-1} \frac{\partial u_{n}}{\partial \theta}\right|^{2} d x \\
& \quad \leq \int_{D_{\delta} \backslash D_{r_{n} R}}-\Delta u_{n}\left(u_{n}-u_{n}^{*}\right) d x+\int_{\partial D_{\delta}} \frac{\partial u_{n}}{\partial r}\left(u_{n}-u_{n}^{*}\right)-\int_{\partial D_{r_{n} R}} \frac{\partial u_{n}}{\partial r}\left(u_{n}-u_{n}^{*}\right) \\
& \quad \leq C(\epsilon+\delta)\left(\int_{D_{\delta} \backslash D_{r_{n} R}}\left(\left|\nabla u_{n}\right|^{2}+\left|\nabla v_{n}\right|^{2}\right) d x+\int_{\partial D_{\delta}}\left|\frac{\partial u_{n}}{\partial r}\right|+\int_{\partial D_{r_{n} R}}\left|\frac{\partial u_{n}}{\partial r}\right|\right) .
\end{aligned}
$$


By Lemma 2.1 and the trace theory, we obtain for the boundary term in (3.15) that

$$
\begin{aligned}
\int_{\partial D_{\delta}}\left|\frac{\partial u_{n}}{\partial r}\right| & \leq C\left(\left\|\nabla u_{n}\right\|_{L^{2}\left(D_{\frac{3}{2} \delta} \backslash D_{\delta}\right)}+\delta\left\|\nabla^{2} u_{n}\right\|_{L^{2}\left(D_{\frac{3}{2} \delta} \backslash D_{\delta}\right)}\right) \\
& \leq C\left(E^{\frac{1}{2}}\left(u_{n}, v_{n} ; D_{2 \delta} \backslash D_{\frac{\delta}{2}}\right)+\delta\left\|\left(\tau_{n}, \kappa_{n}\right)\right\|_{L^{2}\left(D_{2 \delta} \backslash D_{\frac{\delta}{2}}\right)}\right) \\
& \leq C(\epsilon+\delta) .
\end{aligned}
$$

Similarly,

$$
\int_{\partial D_{r_{n} R}}\left|\frac{\partial u_{n}}{\partial r}\right| \leq C(\epsilon+\delta) .
$$

Combining these, we have

$$
\int_{D_{\delta} \backslash D_{r_{n} R}}\left|r^{-1} \frac{\partial u_{n}}{\partial \theta}\right|^{2} d x \leq C(\epsilon+\delta) .
$$

Similarly, we can obtain that

$$
\int_{D_{\delta} \backslash D_{r_{n} R}}\left|r^{-1} \frac{\partial v_{n}}{\partial \theta}\right|^{2} d x \leq C(\epsilon+\delta) .
$$

Without loss of generality, we may assume $\delta=2^{m_{n}} r_{n} R$, where $m_{n}$ is a positive integer which tends to $\infty$ as $n \rightarrow \infty$. By Corollary 2.4 , for $i=0,1, \ldots, m_{n}-1$, we have

$$
\begin{aligned}
& \int_{D_{2^{i+1} r_{n} R} \backslash D_{2^{i} r_{n} R}}\left(\left|\frac{\partial u_{n}}{\partial r}\right|^{2}-\beta\left(u_{n}\right)\left|\frac{\partial v_{n}}{\partial r}+\omega_{j} \frac{\partial u_{n}^{j}}{\partial r}\right|^{2}\right) d x \\
& \leq C\left(\int_{D_{2^{i+1} r_{n} R} \backslash D_{2^{i} r_{n} R}}\left|r^{-1} \frac{\partial u_{n}}{\partial \theta}\right|^{2} d x+\int_{D_{2^{i+1} r_{n} R} \backslash D_{2^{i} r_{n} R}}\left|r^{-1} \frac{\partial v_{n}}{\partial \theta}\right|^{2} d x+2^{i} r_{n} R\right) .
\end{aligned}
$$

Since

$$
\sum_{i=0}^{m_{n}-1} 2^{i} r_{n} R=2^{m_{n}} r_{n} R=\delta
$$

we get

$$
\begin{aligned}
& \int_{D_{\delta} \backslash D_{r_{n} R}}\left(\left|\frac{\partial u_{n}}{\partial r}\right|^{2}-\beta\left(u_{n}\right)\left|\frac{\partial v_{n}}{\partial r}+\omega_{j} \frac{\partial u_{n}^{j}}{\partial r}\right|^{2}\right) d x \\
& =\sum_{i=0}^{m_{n}-1} \int_{D_{2^{i+1} r_{n} R} \backslash D_{2^{i} r_{n} R}}\left(\left|\frac{\partial u_{n}}{\partial r}\right|^{2}-\beta\left(u_{n}\right)\left|\frac{\partial v_{n}}{\partial r}+\omega_{j} \frac{\partial u_{n}^{j}}{\partial r}\right|^{2}\right) d x \leq C(\epsilon+\delta),
\end{aligned}
$$

from which (3.9) follows immediately.

When the 1 -form $\omega \equiv 0$, the behavior of the sequence at the blow-up points is clearer. In fact, we can get identities for the positive energy $E$ instead of for the Lorentzian energy $E_{g}$ and there is no neck between the limit map and the bubbles. More precisely, we have

Lemma 3.3 Assume that $\left\{\left(u_{n}, v_{n}\right)\right\}$ is an approximate Lorentzian harmonic map sequence as in Lemma 3.2 and additionally, we assume that $\omega \equiv 0$ and $\left\|\nabla v_{n}\right\|_{L^{p}} \leq \Lambda$ for some $p>2$, 
then we have that $\sigma^{i}: \mathbb{R}^{2} \cup\{\infty\} \rightarrow N$ is a nontrivial harmonic sphere, $\xi^{i}$ is a constant map and (3.5) becomes

$$
\begin{aligned}
& \lim _{n \rightarrow \infty} E\left(u_{n} ; D_{1}(0)\right)=E\left(u ; D_{1}(0)\right)+\sum_{i=1}^{L} E\left(\sigma^{i}\right), \\
& \lim _{n \rightarrow \infty} E\left(v_{n} ; D_{1}(0)\right)=E\left(v ; D_{1}(0)\right) .
\end{aligned}
$$

Furthermore, The image

$$
u\left(D_{1}(0)\right) \cup \bigcup_{i=1}^{L} \sigma^{i}\left(\mathbb{R}^{2}\right)
$$

is a connected set.

Proof Similar to the proof of Lemma 3.2, to prove (3.18) and (3.19) is equivalent to proving

$$
\lim _{R \rightarrow \infty} \lim _{\delta \rightarrow 0} \lim _{n \rightarrow \infty} E\left(u_{n}, v_{n} ; D_{\delta}(0) \backslash D_{r_{n} R}(0)\right)=0 .
$$

Since $\left\|\nabla v_{n}\right\|_{L^{p}(D)} \leq \Lambda$ for some $p>2$, we get

$$
\int_{D_{\delta} \backslash D_{r_{n} R}}\left|\nabla v_{n}\right|^{2} d x \leq C \delta^{1-\frac{2}{p}}\left(\int_{D_{\delta} \backslash D_{r_{n} R}}\left|\nabla v_{n}\right|^{p} d x\right)^{\frac{2}{p}} \leq C \delta^{1-\frac{2}{p}} .
$$

Since $\omega \equiv 0,(3.17)$ implies that

$$
\int_{D_{\delta} \backslash D_{r_{n} R}}\left|\frac{\partial u_{n}}{\partial r}\right|^{2} d x \leq C \int_{D_{\delta} \backslash D_{r_{n} R}}\left|\nabla v_{n}\right|^{2} d x+C(\epsilon+\delta) .
$$

Combining (3.16), (3.22) with (3.23), we can get (3.21).

To prove (3.20) is equivalent to prove

$$
\lim _{R \rightarrow \infty} \lim _{\delta \rightarrow 0} \lim _{n \rightarrow \infty}\left\|u_{n}\right\|_{o s c}\left(D_{\delta}(0) \backslash D_{r_{n} R}(0)\right)=0 .
$$

To prove (3.24), denote $Q(s):=D_{2^{s_{0}+s}} r_{n} R \backslash D_{2^{s_{0}-s}} r_{n} R$ and consider

$$
f(s):=\int_{Q(s)}\left|\nabla u_{n}\right|^{2} d x
$$

where $0 \leq s_{0} \leq m_{n}$ and $0 \leq s \leq \min \left\{s_{0}, m_{n}-s_{0}\right\}$. Integrating by parts, we get

$$
\begin{aligned}
& \int_{Q(s)}-\Delta u_{n}\left(u_{n}-u_{n}^{*}\right) d x \\
& \quad=\int_{Q(s)} \nabla u_{n} \nabla\left(u_{n}-u_{n}^{*}\right) d x-\int_{\partial Q(s)} \frac{\partial u_{n}}{\partial r}\left(u_{n}-u_{n}^{*}\right) \\
& \quad \geq \frac{1}{2} \int_{Q(s)}\left|\nabla u_{n}\right|^{2} d x+\int_{Q(s)}\left(\frac{1}{2}\left|\nabla u_{n}\right|^{2}-\left|\frac{\partial u_{n}}{\partial r}\right|^{2}\right) d x-\int_{\partial Q(s)} \frac{\partial u_{n}}{\partial r}\left(u_{n}-u_{n}^{*}\right) .
\end{aligned}
$$


By (3.12) and (3.14), we obtain

$$
\begin{aligned}
& \left(\frac{1}{2}-C(\epsilon+\delta)\right) \int_{Q(s)}\left|\nabla u_{n}\right|^{2} d x \\
& \leq \int_{Q(s)}\left(\left|\frac{\partial u_{n}}{\partial r}\right|^{2}-\frac{1}{2}\left|\nabla u_{n}\right|^{2}\right) d x+C(\epsilon+\delta) \int_{Q(s)}\left|\nabla v_{n}\right|^{2} d x \\
& +\int_{\partial D_{2^{s} 0+s_{r_{n} R}}} \frac{\partial u_{n}}{\partial r}\left(u_{n}-u_{n}^{*}\right)-\int_{\partial D_{2^{s_{0}-s_{r_{n} R}}}} \frac{\partial u_{n}}{\partial r}\left(u_{n}-u_{n}^{*}\right) .
\end{aligned}
$$

We deduce from Corollary 2.4 that

$$
\begin{aligned}
\int_{Q(s)}\left(\left|\frac{\partial u_{n}}{\partial r}\right|^{2}-\frac{1}{2}\left|\nabla u_{n}\right|^{2}\right) d x & \leq C \int_{Q(s)}\left|\nabla v_{n}\right|^{2} d x+C 2^{s_{0}+s} r_{n} R \\
& \leq C\left(2^{s_{0}+s} r_{n} R\right)^{1-\frac{2}{p}}\left(\int_{Q(s)}\left|\nabla v_{n}\right|^{p} d x\right)^{\frac{2}{p}}+C 2^{s_{0}+s} r_{n} R \\
& \leq C\left(2^{s_{0}+s} r_{n} R\right)^{1-\frac{2}{p}}
\end{aligned}
$$

For the boundary term in (3.25), by Hölder's inequality and Poincare's inequality, we have

$$
\begin{aligned}
& \left|\int_{\partial D_{2} s_{0}+s_{r_{n} R}} \frac{\partial u_{n}}{\partial r}\left(u_{n}-u_{n}^{*}\right)\right| \leq\left(\int_{\partial D_{2^{s_{0}}+s_{r_{n} R}}}\left|\frac{\partial u_{n}}{\partial r}\right|^{2}\right)^{\frac{1}{2}}\left(\int_{\partial D_{2^{s}+s_{r_{n} R}}}\left|u_{n}-u_{n}^{*}\right|^{2}\right)^{\frac{1}{2}}
\end{aligned}
$$

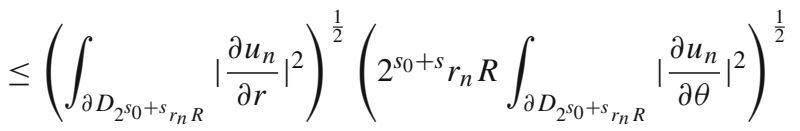

$$
\begin{aligned}
& \leq C 2^{s_{0}+s} r_{n} R \int_{\partial D_{2^{s}+s_{r_{n} R}}}\left|\nabla u_{n}\right|^{2} \text {. }
\end{aligned}
$$

Similarly, we also have

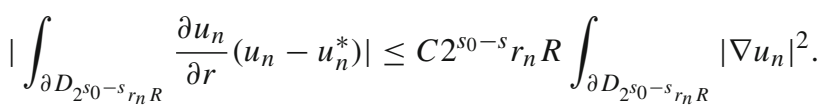

Taking $\epsilon$ and $\delta$ sufficiently small, we get from (3.25) that

$$
f(s) \leq \frac{C}{\ln 2} f^{\prime}(s)+C\left(2^{s_{0}+s} r_{n} R\right)^{1-\frac{2}{p}},
$$

which implies

$$
\left(2^{-\frac{s}{C}} f(s)\right)^{\prime} \geq-C\left(2^{s_{0}} r_{n} R\right)^{1-\frac{2}{p}} 2^{\left(1-\frac{2}{p}-\frac{1}{C}\right) s},
$$

where we can take $C$ sufficiently large such that $1-\frac{2}{p}-\frac{1}{C}>0$. Integrating from 2 to $L$, we arrive at

$$
f(2) \leq C 2^{-\frac{1}{C} L} f(L)+C\left(2^{s_{0}} r_{n} R\right)^{1-\frac{2}{p}} 2^{\left(1-\frac{2}{p}-\frac{1}{C}\right) L} .
$$


Let $s_{0}=i$ and $L=L_{i}:=\min \left\{i, m_{n}-i\right\}$. Noting that $Q\left(L_{i}\right) \subset D_{\delta} \backslash D_{r_{n} R}$, we have

$$
\begin{aligned}
& \int_{D_{2^{i+2} r_{n} R} \backslash D_{2^{i-2} r_{n} R}}\left|\nabla u_{n}\right|^{2} d x \\
& \leq C E\left(u_{n}, D_{\delta} \backslash D_{r_{n} R}\right) 2^{-\frac{1}{C} L_{i}}+C\left(2^{i} r_{n} R\right)^{1-\frac{2}{p}} 2^{\left(1-\frac{2}{p}-\frac{1}{C}\right) L_{i}} \\
& \leq C E\left(u_{n}, D_{\delta} \backslash D_{r_{n} R}\right) 2^{-\frac{1}{C} L_{i}}+C\left(2^{i} r_{n} R\right)^{1-\frac{2}{p}} 2^{\left(1-\frac{2}{p}-\frac{1}{C}\right)\left(m_{n}-i\right)} \\
& \quad=C E\left(u_{n}, D_{\delta} \backslash D_{r_{n} R}\right) 2^{-\frac{1}{C} L_{i}}+C\left(2^{m_{n}} r_{n} R\right)^{1-\frac{2}{p}} 2^{-\frac{1}{C}\left(m_{n}-i\right)} \\
& =C E\left(u_{n}, D_{\delta} \backslash D_{r_{n} R}\right) 2^{-\frac{1}{C} L_{i}}+C \delta^{1-\frac{2}{p}} 2^{\left(-\frac{1}{C}\right)\left(m_{n}-i\right)} \\
& \leq C \epsilon 2^{-\frac{1}{C} i}+C \delta^{1-\frac{2}{p}} 2^{\frac{1}{C}\left(i-m_{n}\right)}
\end{aligned}
$$

where the last inequality follows from the energy identity (3.21). By using Lemma 2.1, now it is easy to deduce (3.24) from (3.22) and the above estimates (3.26) for energy decay.

For the case that the blow-up point is on the boundary of the manifold, the behavior is similar to those in Lemmas 3.2 and 3.3. But the analysis is more complicated. More precisely, we consider an approximate Lorentzian harmonic map sequence $\left\{\left(u_{n}, v_{n}\right)\right\} \in$ $W^{2,2}\left(D_{1}^{+}(0), N \times \mathbb{R}\right)$ with the Dirichlet boundary condition

$$
\left.\left(u_{n}, v_{n}\right)\right|_{\partial^{0} D_{1}^{+}(0)}=(\varphi, \psi) \in C^{2+\alpha}\left(\partial^{0} D_{1}^{+}(0)\right)
$$

for some $0<\alpha<1$ which satisfies that

$$
\left\|\left(\nabla u_{n}, \nabla v_{n}\right)\right\|_{L^{2}\left(D_{1}^{+}(0)\right)}+\left\|\left(\tau_{n}, \kappa_{n}\right)\right\|_{L^{2}\left(D_{1}^{+}(0)\right)} \leq \Lambda .
$$

Without loss of generality, we still suppose that there is only one blow-up point $0 \in D_{1}^{+}(0)$ and the sequence $\left\{\left(u_{n}, v_{n}\right)\right\}$ satisfies that there is some $(u, v)$ such that

$$
\left(u_{n}, v_{n}\right) \rightarrow(u, v) \text { weakly in } W_{l o c}^{2,2}\left(D_{1}^{+}(0) \backslash\{0\}\right) \text { as } n \rightarrow \infty .
$$

For such a sequence, we have

Lemma 3.4 Let $\left\{\left(u_{n}, v_{n}\right)\right\} \in W^{2,2}\left(D_{1}^{+}(0), N \times \mathbb{R}\right)$ be a sequence of approximate Lorentzian harmonic maps satisfying (3.27), (3.28) and (3.29). Up to a subsequence which is still denoted by $\left\{\left(u_{n}, v_{n}\right)\right\}$, we can find a positive integer $L$, points $x_{n}^{i} \in D_{1}^{+}(0)$ and $r_{n}^{i}>0$ satisfying $x_{n}^{i} \rightarrow 0$ and $r_{n}^{i} \rightarrow 0, i=1, \ldots, L$ as $n \rightarrow \infty$ and both of the following two cases may appear during the blow-up process.

(a) If $\frac{\operatorname{dist}\left(x_{n}^{i}, \partial^{0} D_{1}^{+}(0)\right)}{r_{n}^{i}} \rightarrow a^{i}<\infty$, there is a nonconstant Lorentzian harmonic map $\left(\sigma^{i}, \xi^{i}\right)$ : $\mathbb{R}_{a^{i}}^{2} \rightarrow N \times \mathbb{R}$ with a constant boundary condition which is the weak limit of $\left(u_{n}\left(x_{n}^{i}+\right.\right.$ $\left.\left.r_{n}^{i} x\right), v_{n}\left(x_{n}^{i}+r_{n}^{i} x\right)\right)$ in $W_{\text {loc }}^{1,2}\left(\mathbb{R}_{a^{i}}^{2+}\right)$, where

$$
\mathbb{R}_{a^{i}}^{2}:=\left\{\left(x^{1}, x^{2}\right) \in \mathbb{R}^{2} \mid x^{2} \geq a^{i}\right\} \text { and } \mathbb{R}_{a^{i}}^{2+}:=\left\{\left(x^{1}, x^{2}\right) \in \mathbb{R}^{2} \mid x^{2}>a^{i}\right\} ;
$$

(b) If $\frac{\text { dist }\left(x_{n}^{i}, \partial^{0} D_{1}^{+}(0)\right)}{r_{n}^{i}} \rightarrow \infty$, there is a nontrivial Lorentzian harmonic sphere $\left(\sigma^{i}, \xi^{i}\right)$ : $\mathbb{R}^{2} \cup\{\infty\} \rightarrow N \times \mathbb{R}$ which is the weak limit of $\left(u_{n}\left(x_{n}^{i}+r_{n}^{i} x\right), v_{n}\left(x_{n}^{i}+r_{n}^{i} x\right)\right)$ in $W_{l o c}^{1,2}\left(\mathbb{R}^{2}\right)$. 
Furthermore, for both of the two cases, there holds the energy identity

$$
\lim _{n \rightarrow \infty} E_{g}\left(u_{n}, v_{n} ; D_{1}^{+}(0)\right)=E_{g}\left(u, v ; D_{1}^{+}(0)\right)+\sum_{i=1}^{L} E_{g}\left(\sigma^{i}, \xi^{i}\right) .
$$

Here, L just stands for a nonnegative integer which may different from the constant in Lemma 3.2.

Proof Similar to what we have done in the proof of Lemma 3.2, for any $n$, there exist sequences $x_{n} \rightarrow 0$ and $r_{n} \rightarrow 0$ such that

$$
E\left(u_{n}, v_{n} ; D_{r_{n}}^{+}\left(x_{n}\right)\right)=\sup _{\substack{x \in D_{\delta}^{+}, r \leq r_{n} \\ D_{r}^{+}(x) \subset D_{\delta}^{+}}} E\left(u_{n}, v_{n} ; D_{r}^{+}(x)\right)=\frac{1}{8} \min \left\{\epsilon_{1}, \epsilon_{2}\right\},
$$

where $D_{r_{n}}^{+}\left(x_{n}\right):=\left\{\left(y=\left(y^{1}, y^{2}\right) \in \mathbb{R}^{2}|| y-x_{n} \mid \leq r_{n}, y^{2} \geq 0\right)\right\}$. Denote $d_{n}=$ $\operatorname{dist}\left(x_{n}, \partial^{0} D^{+}\right)$. We have that either $\lim \sup _{n \rightarrow \infty} \frac{d_{n}}{r_{n}}<\infty$ or $\lim \sup _{n \rightarrow \infty} \frac{d_{n}}{r_{n}}=\infty$. We discuss these two cases respectively.

Case (a) $\lim \sup _{n \rightarrow \infty} \frac{d_{n}}{r_{n}}<\infty$.

By taking a subsequence, we may assume that $\lim _{n \rightarrow \infty} \frac{d_{n}}{r_{n}}=a \geq 0$. Denote

$$
B_{n}:=\left\{x \in \mathbb{R}^{2} \mid x_{n}+r_{n} x \in D^{+}\right\} .
$$

We have that as $n \rightarrow \infty$,

$$
B_{n} \rightarrow \mathbb{R}_{a}^{2}:=\left\{\left(x^{1}, x^{2}\right) \mid x^{2} \geq-a\right\}
$$

and for any $x \in\left\{x^{2}=-a\right\}$ on the boundary, $x_{n}+r_{n} x \rightarrow 0$.

Define

$$
\widetilde{u}_{n}(x):=u_{n}\left(x_{n}+r_{n} x\right), \widetilde{v}_{n}(x):=v_{n}\left(x_{n}+r_{n} x\right) .
$$

It is easy to get that $\left(\widetilde{u}_{n}, \widetilde{v}_{n}\right): B_{n} \rightarrow N \times \mathbb{R}$ is an approximate Lorentzian harmonic map with $\left(\widetilde{\tau}_{n}, \widetilde{\kappa}_{n}\right)=r_{n}^{2}\left(\tau_{n}, \kappa_{n}\right)$ and

$$
\left(\widetilde{u}_{n}(x), \widetilde{v}_{n}(x)\right)=\left(\varphi\left(x_{n}+r_{n} x\right), \psi\left(x_{n}+r_{n} x\right)\right), \text { if } x_{n}+r_{n} x \in \partial^{0} D^{+} .
$$

Lemma 2.2 and (3.31) tell us that for any $D_{R}(0) \subset \mathbb{R}^{2}$,

$$
\left\|\tilde{u}_{n}\right\|_{W^{2,2}\left(D_{R}(0) \cap B_{n}\right)}+\left\|\widetilde{v}_{n}\right\|_{W^{2,2}\left(D_{R}(0) \cap B_{n}\right)} \leq C\left(\lambda_{1}, \lambda_{2}, \Lambda, R, N\right) .
$$

By a similar argument as in Section 4 of [8], after taking a subsequence of $\left(\widetilde{u}_{n}, \widetilde{v}_{n}\right)$ if necessary (still denoted by $\left(\tilde{u}_{n}, \widetilde{v}_{n}\right)$ ), there is a Lorentzian harmonic map $(\tilde{u}, \widetilde{v}) \in W^{1,2}\left(\mathbb{R}_{a}^{2}, N \times \mathbb{R}\right)$ with the constant boundary condition $\left.(\tilde{u}, \widetilde{v})\right|_{\partial \mathbb{R}_{a}^{2}}=(\phi(0), \psi(0))$ such that, for any $R>0$,

$$
\begin{aligned}
\lim _{n \rightarrow \infty}\left\|\nabla \widetilde{u}_{n}\right\|_{L^{2}\left(D_{R}(0) \cap B_{n}\right)} & =\|\nabla \widetilde{u}\|_{L^{2}\left(D_{R}(0) \cap \mathbb{R}_{a}^{2}\right)}, \\
\lim _{n \rightarrow \infty}\left\|\nabla \widetilde{v}_{n}\right\|_{L^{2}\left(D_{R}(0) \cap B_{n}\right)} & =\|\nabla \widetilde{v}\|_{L^{2}\left(D_{R}(0) \cap \mathbb{R}_{a}^{2}\right)} .
\end{aligned}
$$

Moreover, since $E\left(\tilde{u}, \widetilde{v} ; D_{1}(0) \cap \mathbb{R}_{a}^{2}\right)=\frac{1}{8} \min \left\{\epsilon_{1}, \epsilon_{2}\right\},(\tilde{u}, \widetilde{v})$ is a nontrivial Lorentzian harmonic map with constant boundary $(\phi(0), \psi(0))$.

Case (b) $\lim \sup _{n \rightarrow \infty} \frac{d_{n}}{r_{n}}=\infty$. 
In this case, $\left(\widetilde{u}_{n}, \widetilde{v}_{n}\right)$ lives in $B_{n}$ which tends to $\mathbb{R}^{2}$ as $n \rightarrow \infty$. Moreover, for any $x \in \mathbb{R}^{2}$, when $n$ is sufficiently large, by (3.31), we have

$$
E\left(\widetilde{u}_{n}, \widetilde{v}_{n} ; D_{1}(x)\right) \leq \frac{\epsilon_{1}}{8} .
$$

According to Lemma 2.1, there exist a subsequence of $\left(\widetilde{u}_{n}, \widetilde{v}_{n}\right)$ which is still denoted by $\left.\left(\widetilde{u}_{n}, \widetilde{v}_{n}\right)\right)$ and a Lorentzian harmonic map $(\widetilde{u}(x), \widetilde{v}(x)) \in W^{1,2}\left(\mathbb{R}^{2}, N \times \mathbb{R}\right)$ such that

$$
\lim _{n \rightarrow \infty}\left(\widetilde{u}_{n}(x), \widetilde{v}_{n}(x)\right)=(\widetilde{u}(x), \widetilde{v}(x)) \text { in } W_{l o c}^{1,2}\left(\mathbb{R}^{2}\right) .
$$

By Theorem 2.8, $(\tilde{u}, \widetilde{v})$ can be extended to a Lorentzian harmonic sphere and (3.31) tells us that it is nontrivial.

We call the Lorentzian harmonic map $(\tilde{u}, \widetilde{v})$ obtained in these two cases the first bubble. Without loss of generality, we assume that there is only one bubble at the blow-up point $0 \in D_{1}^{+}(0)$. Under this assumption, similar to (3.10), we have that, for any $\epsilon>0$, there exist constants $\delta>0$ and $R>0$ such that

$$
E\left(u_{n}, v_{n} ; D_{4 \rho}^{+}\left(x_{n}\right) \backslash D_{\frac{\rho}{2}}^{+}\left(x_{n}\right)\right) \leq \epsilon^{2} \text { for any } \rho \in\left[r_{n} R, \frac{\delta}{2}\right]
$$

when $n$ is large enough.

Now to prove the energy identity (3.30) is equivalent to prove

$$
\lim _{R \rightarrow \infty} \lim _{\delta \rightarrow 0} \lim _{n \rightarrow \infty} E_{g}\left(u_{n}, v_{n} ; D_{\delta}^{+}\left(x_{n}\right) \backslash D_{r_{n} R}^{+}\left(x_{n}\right)\right)=0 .
$$

We shall prove (3.33) for the two cases respectively.

For case (a) $\lim _{n \rightarrow \infty} \frac{d_{n}}{r_{n}}=a<\infty$.

For $n$ and $R$ are sufficiently large, we decompose the neck domain $D_{\delta}^{+}\left(x_{n}\right) \backslash D_{r_{n} R}^{+}\left(x_{n}\right)$ into three parts which follows the decomposition in $[15,16]$.

$$
\begin{aligned}
D_{\delta}^{+}\left(x_{n}\right) \backslash D_{r_{n} R}^{+}\left(x_{n}\right) & =D_{\delta}^{+}\left(x_{n}\right) \backslash D_{\frac{\delta}{2}}^{+}\left(x_{n}^{\prime}\right) \cup D_{\frac{\delta}{2}}^{+}\left(x_{n}^{\prime}\right) \backslash D_{2 r_{n} R}^{+}\left(x_{n}^{\prime}\right) \cup D_{2 r_{n} R}^{+}\left(x_{n}^{\prime}\right) \backslash D_{r_{n} R}^{+}\left(x_{n}\right) \\
& :=\Omega_{1} \cup \Omega_{2} \cup \Omega_{3} .
\end{aligned}
$$

Here $x_{n}^{\prime} \in \partial^{0} D^{+}$is the projection of $x_{n}$, i.e. $d_{n}=\left|x_{n}-x_{n}^{\prime}\right|$.

Since $\lim _{n \rightarrow \infty} \frac{d_{n}}{r_{n}}=a$, when $n$ and $R$ are large enough, it is easy to get that

$$
\Omega_{1} \subset D_{\delta}^{+}\left(x_{n}\right) \backslash D_{\frac{\delta}{4}}^{+}\left(x_{n}\right) \text { and } \Omega_{3} \subset D_{4 r_{n} R}^{+}\left(x_{n}\right) \backslash D_{r_{n} R}^{+}\left(x_{n}\right) .
$$

Moreover, for any $\rho \in\left[r_{n} R, \frac{\delta}{2}\right]$, there holds

$$
D_{2 \rho}^{+}\left(x_{n}^{\prime}\right) \backslash D_{\rho}^{+}\left(x_{n}^{\prime}\right) \subset D_{4 \rho}^{+}\left(x_{n}\right) \backslash D_{\rho / 2}^{+}\left(x_{n}\right) .
$$

Then we get from (3.32) that

$$
E\left(u_{n}, v_{n} ; \Omega_{1}\right)+E\left(u_{n}, v_{n} ; \Omega_{3}\right) \leq \epsilon^{2}
$$

and

$$
E\left(u_{n}, v_{n} ; D_{2 \rho}^{+}\left(x_{n}^{\prime}\right) \backslash D_{\rho}^{+}\left(x_{n}^{\prime}\right)\right) \leq \epsilon^{2} \text { for any } \rho \in\left[r_{n} R, \frac{\delta}{2}\right]
$$


By Lemma 2.1, we have

$$
\begin{aligned}
& \left\|u_{n}\right\|_{o s c\left(D_{2 \rho}^{+}\left(x_{n}^{\prime}\right) \backslash D_{\rho}^{+}\left(x_{n}^{\prime}\right)\right)}+\left\|v_{n}\right\|_{o s c\left(D_{2 \rho}^{+}\left(x_{n}^{\prime}\right) \backslash D_{\rho}^{+}\left(x_{n}^{\prime}\right)\right)} \\
& \quad \leq C\left(\left\|\left(\nabla u_{n}, \nabla v_{n}\right)\right\|_{L^{2}\left(D_{4 \rho}^{+}\left(x_{n}^{\prime}\right) \backslash D_{\rho / 2}^{+}\left(x_{n}^{\prime}\right)\right)}+\|(\nabla \phi, \nabla \psi)\|_{L^{2}\left(D_{4 \rho}^{+}\left(x_{n}^{\prime}\right) \backslash D_{\rho / 2}^{+}\left(x_{n}^{\prime}\right)\right)}\right. \\
& \left.\quad+\rho\left\|\left(\nabla^{2} \varphi, \nabla^{2} \psi\right)\right\|_{L^{2}\left(D_{4 \rho}^{+}\left(x_{n}^{\prime}\right) \backslash D_{\rho / 2}^{+}\left(x_{n}^{\prime}\right)\right)}+\rho\left\|\left(\tau_{n}, \kappa_{n}\right)\right\|_{L^{2}\left(D_{4 \rho}^{+}\left(x_{n}^{\prime}\right) \backslash D_{\rho / 2}^{+}\left(x_{n}^{\prime}\right)\right)}\right)
\end{aligned}
$$

for any $\rho \in\left[r_{n} R, \frac{\delta}{2}\right]$.

To estimate the energy concentration in $\Omega_{2}$, we define $\mu_{n}(x):=u_{n}(x)-\varphi(x)$ for $x \in \Omega_{2}$ and

$$
\widehat{\mu}_{n}(x):= \begin{cases}\mu_{n}(x) & x \in \Omega_{2}, \\ -\mu_{n}\left(x^{\prime}\right) & x \in \widehat{\Omega}_{2} \backslash \Omega_{2},\end{cases}
$$

where $\widehat{\Omega}_{2}:=D_{\frac{\delta}{2}}\left(x_{n}^{\prime}\right) \backslash D_{2 r_{n} R}\left(x_{n}^{\prime}\right), x=\left(x^{1}, x^{2}\right)$ and $x^{\prime}=\left(x^{1},-x^{2}\right)$. It is easy to get that $\widehat{\mu}_{n}(x) \in W^{2,2}\left(\widehat{\Omega}_{2}^{2}\right)$ and satisfies

$$
\left|\Delta \widehat{\mu}_{n}(x)\right| \leq \begin{cases}C\left(\left|\nabla u_{n}(x)\right|^{2}+\left|\nabla v_{n}(x)\right|^{2}\right)+\left|\tau_{n}(x)\right|+|\Delta \varphi(x)|, & x \in \Omega_{2}, \\ C\left(\left|\nabla u_{n}\left(x^{\prime}\right)\right|^{2}+\left|\nabla v_{n}\left(x^{\prime}\right)\right|^{2}\right)+\left|\tau_{n}\left(x^{\prime}\right)\right|+\left|\Delta \varphi\left(x^{\prime}\right)\right|, & x \in \widehat{\Omega}_{2} \backslash \Omega_{2} .\end{cases}
$$

Define

$$
\widehat{\mu}_{n}^{*}(r):=\frac{1}{2 \pi} \int_{0}^{2 \pi} \widehat{\mu}_{n}(r, \theta) d \theta,
$$

where $(r, \theta)$ is the polar coordinates at $x_{n}^{\prime}$. By (3.35), we have

$$
\begin{aligned}
\left\|\widehat{\mu}_{n}(x)-\widehat{\mu}_{n}^{*}(x)\right\|_{L^{\infty}\left(\widehat{\Omega}_{2}\right)} & \leq \sup _{r_{n} R \leq \rho \leq \frac{\delta}{2}}\left\|\widehat{\mu}_{n}(x)\right\|_{o s c\left(D_{2 \rho}\left(x_{n}^{\prime}\right) \backslash D_{\rho}\left(x_{n}^{\prime}\right)\right)} \\
& \leq 2 \sup _{r_{n} R \leq \rho \leq \frac{\delta}{2}}\left\|\mu_{n}(x)\right\|_{o s c\left(D_{2 \rho}^{+}\left(x_{n}^{\prime}\right) \backslash D_{\rho}^{+}\left(x_{n}^{\prime}\right)\right)} \\
& \leq C(\epsilon+\delta) .
\end{aligned}
$$

Similar to the proof of (3.15), we can obtain

$$
\begin{aligned}
& \int_{\widehat{\Omega}_{2}}\left|r^{-1} \frac{\partial \widehat{\mu}_{n}}{\partial \theta}\right|^{2} d x \\
& \quad \leq C(\epsilon+\delta)\left(\int_{\widehat{\Omega}_{2}}\left|\Delta \widehat{\mu}_{n}\right| d x+\int_{\partial D_{\frac{\delta}{2}}\left(x_{n}^{\prime}\right)}\left|\frac{\partial \widehat{\mu}_{n}}{\partial r}\right|+\int_{\partial D_{2 r_{n} R}\left(x_{n}^{\prime}\right)}\left|\frac{\partial \widehat{\mu}_{n}}{\partial r}\right|\right) .
\end{aligned}
$$

By direct computations, one can get that

$$
\begin{aligned}
\int_{\widehat{\Omega}_{2}}\left|r^{-1} \frac{\partial \widehat{\mu}_{n}}{\partial \theta}\right|^{2} d x & =2 \int_{\Omega_{2}}\left|r^{-1} \frac{\partial \mu_{n}}{\partial \theta}\right|^{2} d x \\
& =2 \int_{\Omega_{2}}\left|r^{-1} \frac{\partial u_{n}}{\partial \theta}\right|^{2} d x-4 \int_{\Omega_{2}} r^{-2} \frac{\partial u_{n}}{\partial \theta} \frac{\partial \phi}{\partial \theta} d x+2 \int_{\Omega_{2}}\left|r^{-1} \frac{\partial \phi}{\partial \theta}\right|^{2} d x \\
& \geq 2 \int_{\Omega_{2}}\left|r^{-1} \frac{\partial u_{n}}{\partial \theta}\right|^{2} d x-C \delta
\end{aligned}
$$


and

$$
\begin{aligned}
\int_{\widehat{\Omega}_{2}}\left|\Delta \widehat{\mu}_{n}\right| d x & \leq C \int_{\Omega_{2}}\left(\left|\nabla u_{n}\right|^{2}+\left|\nabla v_{n}\right|^{2}\right) d x+\int_{\widehat{\Omega}_{2}}\left(\left|\tau_{n}\right|+|\Delta \phi|\right) d x \\
& \leq C \int_{\Omega_{2}}\left(\left|\nabla u_{n}\right|^{2}+\left|\nabla v_{n}\right|^{2}\right) d x+C \delta .
\end{aligned}
$$

For the boundary terms of the right hand side of (3.36), by the trace theory and Lemma 2.2, we have

$$
\begin{aligned}
& \int_{\partial D_{\delta / 2}\left(x_{n}^{\prime}\right)}\left|\frac{\partial \widehat{\mu}_{n}}{\partial r}\right|=2 \int_{\partial^{+} D_{\delta / 2}\left(x_{n}^{\prime}\right)}\left|\frac{\partial \widehat{\mu}_{n}}{\partial r}\right| \leq C \int_{\partial^{+} D_{\delta / 2}\left(x_{n}^{\prime}\right)}\left(\left|\nabla u_{n}\right|+|\nabla \varphi|\right) \\
& \leq C\left(\left\|\left(\nabla u_{n}, \nabla v_{n}\right)\right\|_{L^{2}\left(D_{\delta}^{+}\left(x_{n}^{\prime}\right) \backslash D_{\frac{1}{4} \delta}^{+}\left(x_{n}^{\prime}\right)\right)}+\delta\left\|\left(\nabla^{2} u_{n}, \nabla^{2} v_{n}\right)\right\|_{L^{2}\left(D_{\delta}^{+}\left(x_{n}^{\prime}\right) \backslash D_{\frac{1}{4} \delta}^{+}\left(x_{n}^{\prime}\right)\right)}+\delta\right) \\
& \leq C\left(\left\|\left(\nabla u_{n}, \nabla v_{n}\right)\right\|_{L^{2}\left(D_{\frac{4}{3} \delta}^{+}\left(x_{n}\right) \backslash D_{\frac{1}{6} \delta}^{+}\left(x_{n}\right)\right)}+\|(\nabla \phi, \nabla \psi)\|_{L^{2}\left(D_{\frac{4}{3} \delta}^{+}\left(x_{n}\right) \backslash D_{\frac{1}{6} \delta}^{+}\left(x_{n}\right)\right)}\right. \\
& \left.\quad+\delta\left\|\left(\nabla^{2} \phi, \nabla^{2} \psi\right)\right\|_{L^{2}\left(D_{\frac{4}{3} \delta}^{+}\left(x_{n}\right) \backslash D_{\frac{1}{6} \delta}^{+}\left(x_{n}\right)\right)}+\delta\left\|\left(\tau_{n}, \kappa_{n}\right)\right\|_{L^{2}\left(D_{\frac{4}{3} \delta}^{+}\left(x_{n}\right) \backslash D_{\frac{1}{6} \delta}^{+}\left(x_{n}\right)\right)}+\delta\right) \\
& \leq C(\epsilon+\delta) .
\end{aligned}
$$

Similarly, we have

$$
\int_{\partial D_{2 r_{n} R}\left(x_{n}^{\prime}\right)}\left|\frac{\partial \widehat{\mu}_{n}}{\partial r}\right| \leq C(\epsilon+\delta) .
$$

Combining these two estimates with (3.36), (3.37) and (3.38), we get

$$
\int_{\Omega_{2}}\left|r^{-1} \frac{\partial u_{n}}{\partial \theta}\right|^{2} d x \leq C(\epsilon+\delta) .
$$

Similarly, we have

$$
\int_{\Omega_{2}}\left|r^{-1} \frac{\partial v_{n}}{\partial \theta}\right|^{2} d x \leq C(\epsilon+\delta) .
$$

By Corollary 2.6, we have

$$
\begin{aligned}
& \int_{D_{2^{i+1} r_{n} R}^{+}\left(x_{n}^{\prime}\right) \backslash D_{2^{i} r_{n} R}^{+}\left(x_{n}^{\prime}\right)}\left(\left|\frac{\partial u_{n}}{\partial r}\right|^{2}-\beta\left(u_{n}\right)\left|\frac{\partial v_{n}}{\partial r}+\omega_{j} \frac{\partial u_{n}^{j}}{\partial r}\right|^{2}\right) d x \\
& \leq C \int_{D_{2^{i+1} r_{n} R}^{+}\left(x_{n}^{\prime}\right) \backslash D_{2^{i} r_{n} R}^{+}\left(x_{n}^{\prime}\right)}\left|r^{-1} \frac{\partial u_{n}}{\partial \theta}\right|^{2} d x \\
& +C \int_{D_{2^{i+1} r_{n} R}^{+}\left(x_{n}^{\prime}\right) \backslash D_{2^{i} r_{n} R}^{+}\left(x_{n}^{\prime}\right)}\left|r^{-1} \frac{\partial v_{n}}{\partial \theta}\right|^{2} d x+2^{i} r_{n} R
\end{aligned}
$$

Thus, we arrive at

$$
\begin{aligned}
& \int_{\Omega_{2}}\left(\left|\frac{\partial u_{n}}{\partial r}\right|^{2}-\beta\left(u_{n}\right)\left|\frac{\partial v_{n}}{\partial r}+\omega_{j} \frac{\partial u_{n}^{j}}{\partial r}\right|^{2}\right) d x \\
& =\sum_{i=0}^{m_{n}-1} \int_{D_{2^{i+1} r_{n} R}^{+}\left(x_{n}^{\prime}\right) \backslash D_{2^{i} r_{n} R}^{+}\left(x_{n}^{\prime}\right)}\left(\left|\frac{\partial u_{n}}{\partial r}\right|^{2}-\beta\left(u_{n}\right)\left|\frac{\partial v_{n}}{\partial r}+\omega_{j} \frac{\partial u_{n}^{j}}{\partial r}\right|^{2}\right) d x \\
& \leq C(\epsilon+\delta) .
\end{aligned}
$$

Then (3.39), (3.40), (3.41), (3.42) and (3.34) imply (3.33). 
For case (b) $\lim _{n \rightarrow \infty} \frac{d_{n}}{r_{n}}=\infty$.

The result for this case can be derived from case (a) and Lemma 3.2. In fact, in this case, for $n$ sufficiently large, we decompose the neck domain $D_{\delta}^{+}\left(x_{n}\right) \backslash D_{r_{n} R}^{+}\left(x_{n}\right)$ as in $[15,16]$ as follows

$$
\begin{aligned}
D_{\delta}^{+}\left(x_{n}\right) \backslash D_{r_{n} R}^{+}\left(x_{n}\right)= & D_{\delta}^{+}\left(x_{n}\right) \backslash D_{\frac{\delta}{2}}^{+}\left(x_{n}^{\prime}\right) \cup D_{\frac{\delta}{2}}^{+}\left(x_{n}^{\prime}\right) \backslash D_{2 d_{n}}^{+}\left(x_{n}^{\prime}\right) \\
& \cup D_{2 d_{n}}^{+}\left(x_{n}^{\prime}\right) \backslash D_{d_{n}}^{+}\left(x_{n}\right) \cup D_{d_{n}}^{+}\left(x_{n}\right) \backslash D_{r_{n} R}^{+}\left(x_{n}\right) \\
& :=\Omega_{1} \cup \Omega_{2} \cup \Omega_{3} \cup \Omega_{4} .
\end{aligned}
$$

Since $\lim _{n \rightarrow \infty} d_{n}=0$ and $\lim _{n \rightarrow \infty} \frac{d_{n}}{r_{n}}=\infty$, when $n$ is large enough, it is easy to get that

$$
\Omega_{1} \subset D_{\delta}^{+}\left(x_{n}\right) \backslash D_{\frac{\delta}{4}}^{+}\left(x_{n}\right) \text {, and } \Omega_{3} \subset D_{4 d_{n}}^{+}\left(x_{n}\right) \backslash D_{d_{n}}^{+}\left(x_{n}\right) \text {. }
$$

Moreover, for any $\rho \in\left[d_{n}, \frac{\delta}{2}\right]$, there holds

$$
D_{2 \rho}^{+}\left(x_{n}^{\prime}\right) \backslash D_{\rho}^{+}\left(x_{n}^{\prime}\right) \subset D_{4 \rho}^{+}\left(x_{n}\right) \backslash D_{\rho / 2}^{+}\left(x_{n}\right) .
$$

By assumption (3.32), we have

$$
E\left(u_{n} ; \Omega_{1}\right)+E\left(u_{n} ; \Omega_{3}\right) \leq \epsilon^{2}
$$

and

$$
\int_{D_{2 \rho}^{+}\left(x_{n}^{\prime}\right) \backslash D_{\rho}^{+}\left(x_{n}^{\prime}\right)}\left|\nabla u_{n}\right|^{2} d x \leq \epsilon^{2} \text { for any } \rho \in\left(d_{n}, \frac{\delta}{2}\right) .
$$

Noting that $\Omega_{4}=D_{d_{n}}^{+}\left(x_{n}\right) \backslash D_{r_{n} R}^{+}\left(x_{n}\right)=D_{d_{n}}\left(x_{n}\right) \backslash D_{r_{n} R}\left(x_{n}\right)$, by Lemma 3.2, there holds

$$
\lim _{R \rightarrow \infty} \lim _{n \rightarrow 0} E_{g}\left(u_{n}, v_{n} ; D_{d_{n}}\left(x_{n}\right) \backslash D_{r_{n} R}\left(x_{n}\right)\right)=0 .
$$

To estimate the energy concentration in $\Omega_{2}$, we can use the same arguments as for case (a) to get that

$$
E_{g}\left(u_{n}, v_{n} ; \Omega_{2}\right) \leq C(\epsilon+\delta) .
$$

Thus we finish the proof of the lemma.

Similar to Lemma 3.3, when $\omega \equiv 0$, we have

Lemma 3.5 Assume that $\left\{\left(u_{n}, v_{n}\right)\right\}$ is an approximate Lorentzian harmonic map sequence as in Lemma 3.4 and additionally, we assume that $\omega \equiv 0$ and $\left\|\nabla v_{n}\right\|_{L^{p}\left(D^{+}\right)} \leq \Lambda$ for some $p>2$, then case (a) in Lemma 3.4 will not happen and in case (b), we have that $\sigma^{i}: \mathbb{R}^{2} \cup\{\infty\} \rightarrow N$ is a nontrivial harmonic sphere, $\xi^{i}$ is a constant map and (3.30) becomes

$$
\begin{aligned}
& \lim _{n \rightarrow \infty} E\left(u_{n} ; D_{1}^{+}(0)\right)=E\left(u ; D_{1}^{+}(0)\right)+\sum_{i=1}^{L} E\left(\sigma^{i}\right), \\
& \lim _{n \rightarrow \infty} E\left(v_{n} ; D_{1}^{+}(0)\right)=E\left(v ; D_{1}^{+}(0)\right) .
\end{aligned}
$$

Furthermore, the image

$$
u\left(D_{1}^{+}(0)\right) \cup \bigcup_{i=1}^{L} \sigma^{i}\left(\mathbb{R}^{2}\right)
$$

is a connected set. 
Proof We use the same symbols as in Lemma 3.4. First, let us show that if $\omega \equiv 0$, Case (a) will not happen. In fact, since $\widetilde{v}$ satisfies

$$
\operatorname{div}(\beta(\widetilde{u}) \nabla \widetilde{v})=0 \text { in } \mathbb{R}_{a}^{2}
$$

and $\left.\widetilde{v}\right|_{\partial \mathbb{R}_{a}^{2}} \equiv \psi(0), \widetilde{v}$ must be a constant map. Thus, $\tilde{u}$ is a harmonic map from $\mathbb{R}_{a}^{2}$ with constant boundary data $\left.\tilde{u}\right|_{\partial \mathbb{R}_{a}^{2}}=\phi(0)$ which implies that $\tilde{u}$ is a constant map [18]. This is a contradiction with $E\left(\tilde{u}, \tilde{v} ; \mathbb{R}_{a}^{2}\right) \geq \frac{1}{8} \min \left\{\epsilon_{1}, \epsilon_{2}\right\}$.

For case (b), when $\omega \equiv 0$, it is clear that $\widetilde{v}$ satisfies the equation

$$
\operatorname{div}(\beta(\widetilde{u}) \nabla \widetilde{v})=0
$$

in $\mathbb{S}^{2}$ with finite energy $\|\nabla \widetilde{v}\|_{L^{2}\left(\mathbb{S}^{2}\right)} \leq C$ which implies that $\widetilde{v}$ must be a constant map. Therefore $\tilde{u}: \mathbb{S}^{2} \rightarrow N$ is a nontrivial harmonic sphere.

Now to prove the energy identities (3.45) and (3.46) is equivalent to prove

$$
\lim _{R \rightarrow \infty} \lim _{\delta \rightarrow 0} \lim _{n \rightarrow \infty} E\left(u_{n}, v_{n} ; D_{\delta}^{+}\left(x_{n}\right) \backslash D_{r_{n} R}^{+}\left(x_{n}\right)\right)=0 .
$$

To prove the no neck result (3.47) is equivalent to prove

$$
\lim _{R \rightarrow \infty} \lim _{\delta \rightarrow 0} \lim _{n \rightarrow \infty}\left\|u_{n}\right\|_{o s c}\left(D_{\delta}^{+}\left(x_{n}\right) \backslash D_{r_{n} R}^{+}\left(x_{n}\right)\right)=0 .
$$

We decompose the neck domain as (3.43). Since $\lim _{n \rightarrow \infty} d_{n}=0$ and $\lim _{n \rightarrow \infty} \frac{d_{n}}{r_{n}}=\infty$, when $n$ is large enough, it is easy to see that

$$
\Omega_{1} \subset D_{\delta}^{+}\left(x_{n}\right) \backslash D_{\frac{\delta}{4}}^{+}\left(x_{n}\right) \text { and } \Omega_{3} \subset D_{4 d_{n}}^{+}\left(x_{n}\right) \backslash D_{d_{n}}^{+}\left(x_{n}\right) .
$$

By (3.32), we have

$$
E\left(u_{n} ; \Omega_{1}\right)+E\left(u_{n} ; \Omega_{3}\right) \leq \epsilon^{2},
$$

which implies that there is no energy loss on $\Omega_{1} \cup \Omega_{3}$. By Lemmas 2.1, 2.2 and (3.32), we get

$$
\begin{aligned}
\| u_{n} & \|_{O s c}\left(D_{\delta}^{+}\left(x_{n}\right) \backslash D_{\frac{\delta}{4}}^{+}\left(x_{n}^{\prime}\right)\right) \\
\leq & \left\|u_{n}\right\|_{O s c\left(D_{\delta}^{+}\left(x_{n}\right) \backslash D_{\frac{\delta}{5}}^{+}\left(x_{n}\right)\right)} \\
\leq & C\left(\left\|\left(\nabla u_{n}, \nabla v_{n}\right)\right\|_{L^{2}\left(D_{\frac{4 \delta}{3}}^{+}\left(x_{n}\right) \backslash D_{\frac{\delta}{6}}^{+}\left(x_{n}\right)\right)}+\|(\nabla \phi, \nabla \psi)\|_{L^{2}\left(D_{\frac{4 \delta}{3}}^{+}\left(x_{n}\right) \backslash D_{\frac{\delta}{6}}^{+}\left(x_{n}\right)\right)}\right. \\
& \left.+\delta\left\|\left(\nabla^{2} \phi, \nabla^{2} \psi\right)\right\|_{L^{2}\left(D_{\frac{4 \delta}{3}}^{+}\left(x_{n}\right) \backslash D_{\frac{\delta}{6}}^{+}\left(x_{n}\right)\right)}+\delta\left\|\left(\tau_{n}, \kappa_{n}\right)\right\|_{L^{2}\left(D_{\frac{4 \delta}{3}}^{+}\left(x_{n}\right) \backslash D_{\frac{\delta}{6}}^{+}\left(x_{n}\right)\right)}\right) \\
\leq & C(\epsilon+\delta)
\end{aligned}
$$

and

$$
\begin{aligned}
\| u_{n} & \|_{O s c\left(D_{2 d_{n}}^{+}\left(x_{n}^{\prime}\right) \backslash D_{d_{n}}^{+}\left(x_{n}\right)\right)} \\
\leq & \left\|u_{n}\right\|_{O s c\left(D_{3 d_{n}}^{+}\left(x_{n}\right) \backslash D_{d_{n}}^{+}\left(x_{n}\right)\right)} \\
\leq & C\left(\left\|\left(\nabla u_{n}, \nabla v_{n}\right)\right\|_{L^{2}\left(D_{4 d_{n}}^{+}\left(x_{n}\right) \backslash D_{\frac{d_{n}}{2}}^{+}\left(x_{n}\right)\right)}+\|(\nabla \phi, \nabla \psi)\|_{L^{2}\left(D_{4 d_{n}}^{+}\left(x_{n}\right) \backslash D_{\frac{d_{n}}{2}}^{+}\left(x_{n}\right)\right)}\right. \\
& \left.+d_{n}\left\|\left(\nabla^{2} \phi, \nabla^{2} \psi\right)\right\|_{L^{2}\left(D_{4 d_{n}}^{+}\left(x_{n}\right) \backslash D_{\frac{d_{n}}{2}}^{+}\left(x_{n}\right)\right)}+d_{n}\left\|\left(\tau_{n}, \kappa_{n}\right)\right\|_{L^{2}\left(D_{4 d_{n}}^{+}\left(x_{n}\right) \backslash D_{\frac{d_{n}}{2}}^{+}\left(x_{n}\right)\right)}\right) \\
\leq & C(\epsilon+\delta),
\end{aligned}
$$


when $n, R$ are large and $\delta$ is small, which implies that there is no neck on $\Omega_{1} \cup \Omega_{3}$.

Moreover, for any $d_{n} \leq \rho \leq \frac{\delta}{2}$, there holds

$$
D_{2 \rho}^{+}\left(x_{n}^{\prime}\right) \backslash D_{\rho}^{+}\left(x_{n}^{\prime}\right) \subset D_{4 \rho}^{+}\left(x_{n}\right) \backslash D_{\rho / 2}^{+}\left(x_{n}\right) .
$$

when $n$ is big enough, then (3.32) tells us

$$
\int_{D_{2 \rho}^{+}\left(x_{n}^{\prime}\right) \backslash D_{\rho}^{+}\left(x_{n}^{\prime}\right)}\left|\nabla u_{n}\right|^{2} d x \leq \epsilon^{2} \text { for any } \rho \in\left(d_{n}, \frac{\delta}{2}\right) .
$$

Combining this with Lemma 2.2, we get

$$
\begin{aligned}
& \left\|u_{n}\right\|_{o s c\left(D_{2 \rho}^{+}\left(x_{n}^{\prime}\right) \backslash D_{\rho}^{+}\left(x_{n}^{\prime}\right)\right)} \\
& \quad \leq C\left(\left\|\left(\nabla u_{n}, \nabla v_{n}\right)\right\|_{L^{2}\left(D_{4 \rho}^{+}\left(x_{n}^{\prime}\right) \backslash D_{\rho / 2}^{+}\left(x_{n}^{\prime}\right)\right)}+\|(\nabla \phi, \nabla \psi)\|_{L^{2}\left(D_{4 \rho}^{+}\left(x_{n}^{\prime}\right) \backslash D_{\rho / 2}^{+}\left(x_{n}^{\prime}\right)\right)}\right. \\
& \left.\quad+\rho\left\|\left(\nabla^{2} \varphi, \nabla^{2} \psi\right)\right\|_{L^{2}\left(D_{4 \rho}^{+}\left(x_{n}^{\prime}\right) \backslash D_{\rho / 2}^{+}\left(x_{n}^{\prime}\right)\right)}+\rho\left\|\left(\tau_{n}, \kappa_{n}\right)\right\|_{L^{2}\left(D_{4 \rho}^{+}\left(x_{n}^{\prime}\right) \backslash D_{\rho / 2}^{+}\left(x_{n}^{\prime}\right)\right)}\right)
\end{aligned}
$$

for any $\rho \in\left(d_{n}, \frac{\delta}{2}\right)$.

Noting that $\Omega_{4}=D_{d_{n}}^{+}\left(x_{n}\right) \backslash D_{r_{n} R}^{+}\left(x_{n}\right)=D_{d_{n}}\left(x_{n}\right) \backslash D_{r_{n} R}\left(x_{n}\right)$, the proofs of (3.48) and (3.49) are reduced to the case in Lemma 3.3 and we have

$$
\lim _{R \rightarrow \infty} \lim _{n \rightarrow 0} E\left(u_{n}, v_{n} ; D_{d_{n}}\left(x_{n}\right) \backslash D_{r_{n} R}\left(x_{n}\right)\right)=0
$$

and

$$
\lim _{R \rightarrow \infty} \lim _{n \rightarrow 0} \operatorname{osc}\left(u_{n}\right)_{D_{d_{n}}\left(x_{n}\right) \backslash D_{r_{n} R}\left(x_{n}\right)=0 .}
$$

To prove that there is no energy loss on $\Omega_{2}$, noting that $\left\|\nabla v_{n}\right\|_{L^{p}\left(D^{+}\right)} \leq \Lambda$ for some $p>2$, we get

$$
\int_{D_{\delta}^{+}\left(x_{n}\right) \backslash D_{r_{n} R}^{+}\left(x_{n}\right)}\left|\nabla v_{n}\right|^{2} d x \leq C \delta^{1-\frac{2}{p}}\left(\int_{D_{\delta}^{+}\left(x_{n}\right) \backslash D_{r_{n} R}^{+}\left(x_{n}\right)}\left|\nabla v_{n}\right|^{p} d x\right)^{\frac{2}{p}} \leq C \delta^{1-\frac{2}{p}} .
$$

Combining this with (3.44), we obtain

$$
E\left(u_{n}, \Omega_{2}\right) \leq C E\left(v_{n}, \Omega\right)+C(\epsilon+\delta) \leq C\left(\epsilon+\delta^{1-\frac{2}{p}}\right) .
$$

Then, (3.48) follows from (3.50), (3.53), (3.55) and (3.56).

Now we only need to analyze the neck on $\Omega_{2}$.

We denote $Q(s):=D_{2^{s_{0}+s} 2 r_{n} R}^{+}\left(x_{n}^{\prime}\right) \backslash D_{2^{s_{0}-s} 2 r_{n} R}^{+}\left(x_{n}^{\prime}\right)$ and $\widehat{Q}(s):=D_{2^{s_{0}+s} 2 r_{n} R}\left(x_{n}^{\prime}\right) \backslash$ $D_{2^{s} 0^{-s}} 2 r_{n} R\left(x_{n}^{\prime}\right)$, where $0 \leq s_{0} \leq m_{n}$ and $0 \leq s \leq \min \left\{s_{0}, m_{n}-s_{0}\right\}$. Let

$$
f(s):=\int_{Q(s)}\left|\nabla u_{n}\right|^{2} d x .
$$

Similar to the derivation of (3.15), we can obtain

$$
\begin{aligned}
& \int_{\widehat{Q}(s)}\left|\nabla \widehat{\mu}_{n}\right|^{2} d x-\int_{\widehat{Q}(s)}\left|\frac{\partial \widehat{\mu}_{n}}{\partial r}\right|^{2} d x \\
& \quad \leq C(\epsilon+\delta) \int_{\widehat{Q}(s)}\left|\Delta \widehat{\mu}_{n}\right| d x+\int_{\partial \widehat{Q}(s)} \frac{\partial \widehat{\mu}_{n}}{\partial r}\left(\widehat{\mu}_{n}-\widehat{\mu}_{n}^{*}\right) .
\end{aligned}
$$


By direct computations, we obtain

$$
\begin{aligned}
& \int_{\widehat{Q}(s)}\left|\nabla \widehat{\mu}_{n}\right|^{2} d x-\int_{\widehat{Q}(s)}\left|\frac{\partial \widehat{\mu}_{n}}{\partial r}\right|^{2} d x=\int_{Q(s)}\left|\nabla \mu_{n}\right|^{2} d x-2 \int_{Q(s)}\left(\left|\frac{\partial \mu_{n}}{\partial r}\right|^{2}-\frac{1}{2}\left|\nabla \mu_{n}\right|^{2}\right) d x \\
& =\int_{Q(s)}\left|\nabla u_{n}\right|^{2} d x-2 \int_{Q(s)}\left(\left|\frac{\partial u_{n}}{\partial r}\right|^{2}-\frac{1}{2}\left|\nabla u_{n}\right|^{2}\right) d x+4 \int_{Q(s)}\left(\frac{\partial u_{n}}{\partial r} \frac{\partial \phi}{\partial r}-\nabla u_{n} \nabla \phi\right) d x \\
& \quad+2 \int_{Q(s)}\left(|\nabla \phi|^{2}-\left|\frac{\partial \phi}{\partial r}\right|^{2}\right) d x \\
& \geq \int_{Q(s)}\left|\nabla u_{n}\right|^{2} d x-2 \int_{Q(s)}\left(\left|\frac{\partial u_{n}}{\partial r}\right|^{2}-\frac{1}{2}\left|\nabla u_{n}\right|^{2}\right) d x-C 2^{s_{0}+s} r_{n} R .
\end{aligned}
$$

It is easy to check that (3.38) still holds on $\Omega_{2}$. Combining this with $\left\|\nabla v_{n}\right\|_{L^{p}\left(D^{+}\right)} \leq C$, we have

$$
\begin{aligned}
\int_{\widehat{Q}(s)}\left|\Delta \widehat{\mu}_{n}\right| d x & \leq C \int_{Q(s)}\left(\left|\nabla u_{n}\right|^{2}+\left|\nabla v_{n}\right|^{2}\right) d x+C 2^{s_{0}+s} r_{n} R \\
& \leq C \int_{Q(s)}\left|\nabla u_{n}\right|^{2} d x+C\left(2^{s_{0}+s} r_{n} R\right)^{1-\frac{2}{p}} .
\end{aligned}
$$

Then (3.57) implies

$$
\begin{aligned}
(1 & -C(\epsilon+\delta)) \int_{Q(t)}\left|\nabla u_{n}\right|^{2} d x \\
& \leq \int_{\partial \widehat{Q}(s)} \frac{\partial \widehat{\mu}_{n}}{\partial r}\left(\widehat{\mu}_{n}-\widehat{\mu}_{n}^{*}\right)+2 \int_{Q(s)}\left(\left|\frac{\partial u_{n}}{\partial r}\right|^{2}-\frac{1}{2}\left|\nabla u_{n}\right|^{2}\right) d x+C\left(2^{s_{0}+s} r_{n} R\right)^{1-\frac{2}{p}} \\
& \leq \int_{\partial \widehat{Q}(s)} \frac{\partial \widehat{\mu}_{n}}{\partial r}\left(\widehat{\mu}_{n}-\widehat{\mu}_{n}^{*}\right)+C\left(2^{s_{0}+s} r_{n} R\right)^{1-\frac{2}{p}}
\end{aligned}
$$

where the last inequality follows from Corollary 2.6 and (3.22).

For the boundary term on the right hand side of (3.58), by Hölder's inequality and Poincare's inequality, we have

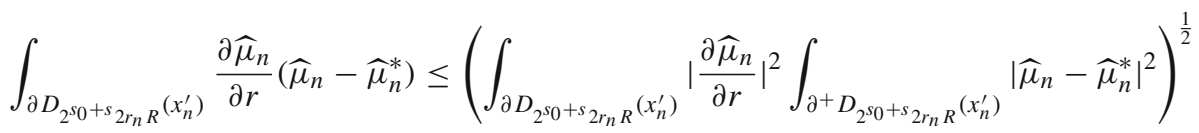

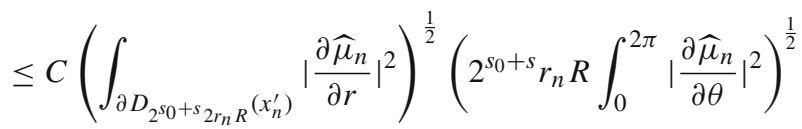

$$
\begin{aligned}
& \leq C 2^{s_{0}+s} d_{n} \int_{\partial D_{2^{s} s^{+}+s_{2 r_{n} R}}\left(x_{n}^{\prime}\right)}\left|\nabla \widehat{\mu}_{n}\right|^{2} \\
& \leq C 2^{s_{0}+s} d_{n} \int_{\partial^{+} D_{2^{s}{ }^{+}+s_{2 r_{n} R}}^{+}\left(x_{n}^{\prime}\right)}\left|\nabla \mu_{n}\right|^{2}
\end{aligned}
$$

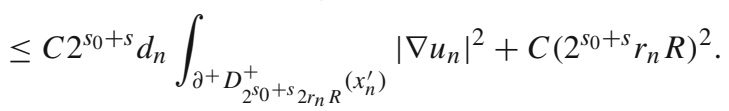

Similarly, we can obtain

$$
\int_{\partial D_{2^{s} 0^{-s} 2 r_{n} R}\left(x_{n}^{\prime}\right)} \frac{\partial \widehat{\mu}_{n}}{\partial r}\left(\widehat{\mu}_{n}-\widehat{\mu}_{n}^{*}\right) \leq C 2^{s_{0}-s} d_{n} \int_{\partial^{+} D_{2^{s} 0^{-s} 2 r_{n} R}^{+}\left(x_{n}^{\prime}\right)}\left|\nabla u_{n}\right|^{2}+C\left(2^{s_{0}-s} r_{n} R\right)^{2} .
$$


Taking $\epsilon$ and $\delta$ sufficiently small, we have

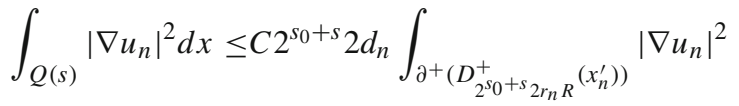

$$
\begin{aligned}
& +C 2^{s_{0}-s} 2 d_{n} \int_{\partial^{+}\left(D_{2^{s_{0}-s_{2}}+r_{n} R}\left(x_{n}^{\prime}\right)\right)}\left|\nabla u_{n}\right|^{2}+C\left(2^{s_{0}+s} r_{n} R\right)^{1-\frac{2}{p}},
\end{aligned}
$$

which gives us

$$
f(s) \leq \frac{C}{\log 2} f^{\prime}(s)+C\left(2^{s_{0}+s} r_{n} R\right)^{1-\frac{2}{p}} .
$$

(3.59) implies that

$$
\left(2^{-\frac{s}{C}} f(s)\right)^{\prime} \geq-C\left(2^{s_{0}} r_{n} R\right)^{1-\frac{2}{p}} 2^{\left(1-\frac{2}{p}-\frac{1}{C}\right) s} .
$$

Integrating from 2 to $L$, we arrive at

$$
f(2) \leq C 2^{-\frac{1}{C} L} f(L)+C\left(2^{s_{0}} r_{n} R\right)^{1-\frac{2}{p}} 2^{\left(1-\frac{2}{p}-\frac{1}{C}\right) L} .
$$

The rest proof is the same as the proof in Lemma 3.3. Thus we finish the analysis of energy loss and no neck property on $\Omega_{1} \cup \Omega_{3}, \Omega_{4}$ and $\Omega_{2}$ and get (3.48) and (3.49).

We can now prove Theorems 1.1 and 1.2.

Proof of Theorems 1.1 and 1.2 Theorem 1.1 is a direct conclusion of Lemmas 3.2 and 3.4.

If $\omega \equiv 0, \widetilde{v}=v-\psi$ satisfies

$$
\operatorname{div}(\beta(u) \nabla \widetilde{v})=-\operatorname{div}(\beta(u) \nabla \psi)+\kappa
$$

with the boundary condition $\left.\widetilde{v}\right|_{\partial M}=0$. By Theorem 1 in [21], for any $1<p<\infty$, we have

$$
\|\nabla \widetilde{v}\|_{L^{p}(M)} \leq C\left(\|\nabla \psi\|_{L^{p}(M)}+\|\kappa\|_{L^{2}(M)}\right) .
$$

Thus we have

$$
\|\nabla v\|_{L^{p}(M)} \leq C\left(\|\nabla \psi\|_{L^{p}(M)}+\|\kappa\|_{L^{2}(M)}\right) .
$$

Then, Theorem 1.2 is a direct conclusion of Lemmas 3.3 and 3.5.

\section{Applications to the Lorentzian harmonic map flow}

At the beginning of this section, let us recall a lemma in [8] which is useful in this part.

Lemma 4.1 (Lemmas 2.1, 2.4 in [8]) Suppose $(u, v) \in \mathcal{V}\left(M_{0}^{T_{1}} ; N \times \mathbb{R}\right)$ is a solution of (1.14) and (1.15), then the Lorentzian energy $E_{g}(u(t), v(t))$ is non-increasing on $\left[0, T_{1}\right)$ and for any $0 \leq s \leq t<T_{1}$, there holds

$$
E_{g}(u(t), v(t))+\int_{s}^{t} \int_{M}\left|\partial_{t} u\right|^{2} d x d t \leq E_{g}(u(s), v(s)) .
$$

Moreover, for any $1<p<\infty, t>0$, there holds

$$
\int_{M}|\nabla u(\cdot, t)|^{2} d x+\int_{M}|\nabla v(\cdot, t)|^{p} d x+\int_{0}^{t} \int_{M}\left|\partial_{t} u\right|^{2} d x d t \leq C\left(p, \lambda_{1}, \lambda_{2}, \phi, \psi\right) .
$$


Lemma 4.2 Let $(u, v) \in \mathcal{V}\left(M_{0}^{T_{1}} ; N \times \mathbb{R}\right)$ be a solution to (1.14) and (1.15). There exists a positive constant $R_{0}<1$ such that, for any $x_{0} \in M, 0 \leq R \leq R_{0}$ and $0<s \leq t<T_{1}$, there hold

$$
E\left(u(t) ; B_{R}^{M}\left(x_{0}\right)\right) \leq E\left(u(s) ; B_{2 R}^{M}\left(x_{0}\right)\right)+C \frac{t-s}{R^{2}},
$$

and

$$
E\left(u(s) ; B_{R}^{M}\left(x_{0}\right)\right) \leq E\left(u(t) ; B_{2 R}^{M}\left(x_{0}\right)\right)+C \frac{t-s}{R^{2}}+C \int_{s}^{t} \int_{M}\left|\partial_{t} u\right|^{2} d x d t,
$$

where $B_{R}^{M}\left(x_{0}\right) \subset M$ is the geodesic ball centered at point $x_{0}$ with radius $R, C$ is a positive constant depending on $\lambda_{1}, \lambda_{2}, M, N, E(\phi),\|\psi\|_{W^{1,4}(M)}$.

Proof Let $\eta \in C_{0}^{\infty}\left(B_{2 R}^{M}\left(x_{0}\right)\right)$ be a cut-off function such that $\eta(x)=\eta\left(\left|x-x_{0}\right|\right), 0 \leq \eta \leq 1$, $\left.\eta\right|_{B_{R}^{M}\left(x_{0}\right)} \equiv 1$ and $|\nabla \eta| \leq \frac{C}{R}$. By direct computations, we get

$$
\begin{aligned}
\frac{d}{d t} \frac{1}{2} \int_{M}|\nabla u|^{2} \eta^{2} & =\int_{M}\left\langle\nabla u, \nabla u_{t}\right\rangle \eta^{2} \\
& =\int_{\partial B_{2 R}^{M}\left(x_{0}\right)} \frac{\partial u}{\partial r} \cdot u_{t} \eta^{2}-\int_{M}\left\langle\Delta u, u_{t}\right\rangle \eta^{2}-2 \int_{M} \nabla u \cdot \nabla \eta \eta u_{t} \\
& =\int_{M}\left\langle-u_{t}-B^{\top}(u)|\nabla v|^{2}, u_{t}\right\rangle \eta^{2}-2 \int_{M} \nabla u \cdot \nabla \eta \eta u_{t} \\
& =-\int_{M}\left|u_{t}\right|^{2} \eta^{2}-\int_{M} B^{\top}(u)|\nabla v|^{2} \cdot u_{t} \eta^{2}-2 \int_{M} \nabla u \cdot \nabla \eta \eta u_{t} .
\end{aligned}
$$

On the one hand, by Lemma 4.1 and Young's inequality, we have

$$
\frac{d}{d t} \frac{1}{2} \int_{M}|\nabla u|^{2} \eta^{2} \leq-\frac{1}{2} \int_{M}\left|u_{t}\right|^{2} \eta^{2}+C \int_{M}|\nabla u|^{2}|\nabla \eta|^{2}+C \int_{M}|\nabla v|^{4} \eta^{2} \leq \frac{C}{R^{2}} .
$$

By integrating the above inequality from $s$ to $t$, we can get (4.1).

On the other hand, by Lemma 4.1 and Young's inequality, we also have

$$
\begin{aligned}
\frac{d}{d t} \frac{1}{2} \int_{M}|\nabla u|^{2} \eta^{2} & \geq-\frac{3}{2} \int_{M}\left|u_{t}\right|^{2} \eta^{2}-C \int_{M}|\nabla u|^{2}|\nabla \eta|^{2}-C \int_{M}|\nabla v|^{4} \eta^{2} \\
& \geq-\frac{3}{2} \int_{M}\left|u_{t}\right|^{2} \eta^{2}-\frac{C}{R^{2}} .
\end{aligned}
$$

Then (4.2) follows immediately from integrating the above inequality from $s$ to $t$.

With the help of Lemma 4.2, we can apply the standard argument (see Lemma 6.4.10 in [20]) to obtain

Lemma 4.3 Let $(u, v) \in \mathcal{V}\left(M_{0}^{T_{1}} ; N \times \mathbb{R}\right)$ be a solution to (1.14) and (1.15). Assume that there is only one singular point $x_{0} \in M$ at time $T_{1}$. Then there exists a positive number $m>0$ such that, as $t \uparrow T_{1}$,

$$
|\nabla u|^{2}(x, t) d x \rightarrow m \delta_{x_{0}}+|\nabla u|^{2}\left(x, T_{1}\right) d x \text { as Radon measures. }
$$

Here $\delta_{x_{0}}$ denotes the $\delta$-mass at $x_{0}$.

Now we shall prove Theorems 1.3 and 1.4. 
Proof of Theorem 1.3 In fact, Theorem 1.3 is a consequence of Lemma 4.1, Theorems 1.1 and 1.2.

By Lemma 4.1, we can find a positive sequence $t_{n} \rightarrow \infty$, such that

$$
\lim _{n \rightarrow \infty} \int_{M}\left|\partial_{t} u\right|^{2}\left(\cdot, t_{n}\right) d x=0 \quad \text { and } \quad E\left(u\left(\cdot, t_{n}\right), v\left(\cdot, t_{n}\right)\right) \leq C .
$$

Taking the sequence to be $\left(u_{n}, v_{n}\right)=\left(u\left(\cdot, t_{n}\right), v\left(\cdot, t_{n}\right)\right)$ with $\left(\tau_{n}, h_{n}\right)=\left(\partial_{t} u\left(\cdot, t_{n}\right), 0\right)$ in Theorems 1.1 and 1.2, the conclusions of Theorem 1.3 follow immediately.

Proof of Theorem 1.4 With the help of Lemmas 4.2, 4.3, Theorems 1.1 and 1.2, the proof of (1.19) is almost the same as the proof for the harmonic map flow and we omit the details here. One can refer to [20] for the interior case and to $[15,16]$ for the boundary case.

It is not hard to prove that there is a unique weak limit $\left(u\left(T_{1}\right), v\left(T_{1}\right)\right) \in W^{1,2}(M, N \times \mathbb{R})$ of $(u(t), v(t))$ in $W^{1,2}(M)$ as $t \rightarrow T_{1}$ (one can refer to the proof of Theorem 1.2 in [14] for a similar argument). Moreover, by Lemma 4.1,

$$
v(t) \rightarrow v\left(T_{1}\right) \text { weakly in } W^{1,4}(M) .
$$

Then, we have

$$
\begin{aligned}
\int_{M} \beta(u(t))|\nabla v(t)|^{2} d x-\int_{M} \beta\left(u\left(T_{1}\right)\right)\left|\nabla v\left(T_{1}\right)\right|^{2} d x \\
=\int_{M} \beta(u(t)) \nabla v(t) \nabla\left(v(t)-v\left(T_{1}\right)\right)+\left(\beta(u(t)) \nabla v(t)-\beta\left(u\left(T_{1}\right)\right) \nabla v\left(T_{1}\right)\right) \nabla v\left(T_{1}\right) d x \\
=\int_{M}\left(\beta(u(t))-\beta\left(u\left(T_{1}\right)\right)\right) \nabla v(t) \nabla v\left(T_{1}\right)+\beta\left(u\left(T_{1}\right)\right)\left(\nabla v(t)-\nabla v\left(T_{1}\right)\right) \nabla v\left(T_{1}\right) d x \\
=\mathbb{I}+\mathbb{I I},
\end{aligned}
$$

where the first term of the second line is zero by integrating by parts and Eq. (1.14). Noting that

$$
\mathbb{I} \leq C\|\nabla v(t)\|_{L^{4}(M)}\left\|\nabla v\left(T_{1}\right)\right\|_{L^{4}(M)}\left\|u(t)-u\left(T_{1}\right)\right\|_{L^{2}(M)},
$$

by weak convergence, we have

$$
\lim _{t \rightarrow T_{1}} \int_{M} \beta(u(t))|\nabla v(t)|^{2} d x=\int_{M} \beta\left(u\left(T_{1}\right)\right)\left|\nabla v\left(T_{1}\right)\right|^{2} d x .
$$

Combining this with (1.19), we get (1.20).

Acknowledgements Open access funding provided by Max Planck Society.

Open Access This article is distributed under the terms of the Creative Commons Attribution 4.0 International License (http://creativecommons.org/licenses/by/4.0/), which permits unrestricted use, distribution, and reproduction in any medium, provided you give appropriate credit to the original author(s) and the source, provide a link to the Creative Commons license, and indicate if changes were made.

\section{References}

1. Alday, L., Maldacena, J.: Null polygonal Wilson loops and minimal surfaces in anti-de-Sitter space. J. High Energy Phys. 082, 59 (2009)

2. Benci, V., Fortunato, D., Giannoni, F.: On the existence of multiple geodesics in static space-times. Ann. Inst. H. Poincaré 8(1), 79-102 (1991) 
3. Bryant, R.: A duality theorem for Willmore surfaces. J. Differ. Geom. 20(1), 23-53 (1984)

4. Chen, Q., Jost, J., Li, J., Wang, G.: Dirac-harmonic maps. Math. Z. 254(2), 409-432 (2006)

5. Ding, W., Tian, G.: Energy identity for a class of approximate harmonic maps from surfaces. Commun. Anal. Geom. 3(4), 543-554 (1995)

6. Greco, C.: The Dirichlet-problem for harmonic maps from the disk in to a lorentzian warped product. Ann. Inst. H. Poincaré 10(2), 239-252 (1993)

7. Greco, C.: A multiplicity result for the Dirichlet problem for harmonic maps from the disk into a Lorentzian warped product. Nonlinear Anal. 28(10), 1661-1668 (1997)

8. Han, X., Jost, J., Liu, L., Zhao, L.: Global Existence of Harmonic Map Heat Flow Into Lorentzian Manifolds. MPI MIS Preprint: 70/2016. https://www.mis.mpg.de/publications/preprints/2016/prepr2016-70. html. Accessed 11 Oct 2016

9. Han, X., Zhao, L., Zhu, M.: Energy identity for harmonic maps into standard stationary Lorentzian manifolds. J. Geom. Phys. 114, 621-630 (2017)

10. Huang, T., Wang, C.: Boundary Bubbling Analysis of Approximate Harmonic Maps Under Either Weak or Strong Anchoring Conditions in Dimension Two. arXiv:1607.05357

11. Isobe, T.: Optimal regularity of harmonic maps from a Riemannian manifold into a static Lorentzian manifold. Pac. J. Math. 178(1), 71-93 (1997)

12. Isobe, T.: Regularity of harmonic maps into a static Lorentzian manifold. J. Geom. Anal. 8(3), 447-463 (1998)

13. Jost, J.: Two-Dimensional Geometric Variational Problems. Wiley, New York (1991)

14. Jost, J., Liu, L., Zhu, M.: A global weak solution of the Dirac-harmonic map flow. Ann. Inst. H. Poincare Anal. Non Lineaire (2017). https://doi.org/10.1016/j.anihpc.2017.01.002

15. Jost, J., Liu, L., Zhu, M.: The Qualitative Behavior at the Free Boundary for Approximate Harmonic Maps from Surfaces. MPI MIS Preprint: 26/2016. https://www.mis.mpg.de/publications/preprints/2016/ prepr2016-26.html. Accessed 21 Mar 2016

16. Jost, J., Liu, L., Zhu, M.: Bubbling Analysis Near the Dirichlet Boundary for Approximate Harmonic Maps from Surfaces. MPI MIS Preprint: 38/2016 (to appear in Comm. Anal. Geom.). https://www.mis. mpg.de/publications/preprints/2016/prepr2016-38.html. Accessed 29 June 2016

17. Kramer, D., Stephani, H., Hertl, E., MacCallum, M.: Exact Solutions of Einsteins Field Equations. Cambridge University Press, Cambridge (1980)

18. Lemaire, L.: Applications harmoniques de surfaces riemanniennes. J. Differ. Geom. 13(1), 51-78 (1978)

19. Li, J., Liu, L.: Partial Regularity of Harmonic Maps from a Riemannian Manifold Into a Lorentzian Manifold. arXiv: 1704.08673

20. Lin, F., Wang, C.: The Analysis of Harmonic Maps and Their Heat Flows, p. xii+267. World Scientific Publishing Co. Pte. Ltd., Hackensack, NJ (2008)

21. Meyers, G.: An $L^{p}$-estimate for the gradient of solutions of second order elliptic divergence equations. Annali Della Scuola Normale superiore di Pisa-Classe di Scienze 17(3), 189-206 (1963)

22. O'Neill, B.: Semi-Riemannian Geometry: With Applications to Relativity Pure and Applied Mathematics, vol. 103, p. xiii+468. Academic Press Inc./Harcourt Brace Jovanovich Publishers, New York (1983)

23. Parker, T.: Bubble tree convergence for harmonic maps. J. Differ. Geom. 44(3), 595-633 (1996)

24. Qing, J., Tian, G.: Bubbling of the heat flows for harmonic maps from surfaces. Commun. Pure Appl. Math. 50(4), 295-310 (1997)

25. Rivière, T.: Conservation laws for conformally invariant variational problems. Invent. Math. 168(1), 1-22 (2007)

26. Rivière, T., Struwe, M.: Partial regularity for harmonic maps and related problems. Commun. Pure Appl. Math. 61(4), 451-463 (2007)

27. Sacks, J., Uhlenbeck, K.: The existence of minimal immersions of 2-spheres. Ann. Math. 113, 1-24 (1981)

28. Sharp, B.: Higher integrability for solutions to a system of critical elliptic PDE. Methods Appl. Anal. 21(2), 221-240 (2014)

29. Sharp, B., Topping, P.: Decay estimates for Riviere's equation, with applications to regularity and compactness. Trans. Am. Math. Soc. 365(5), 2317-2339 (2013)

30. Sharp, B., Zhu, M.: Regularity at the free boundary for Dirac-harmonic maps from surfaces. Calc. Var. Partial Differ. Equ. 55(27), 1-30 (2016)

31. Zhao, L.: Energy identities for Dirac-harmonic maps. Calc. Var. Partial Differ. Equ. 28(1), 121-138 (2007)

32. Zhu, M.: Regularity for harmonic maps into certain pseudo-Riemannian manifolds. J. Math. Pures Appl. 99(1), 106-123 (2013) 